ARTICLE

DOI: $10.1038 / s 41467-017-01756-1$

\title{
Alternative transcription start site selection in Mr-OPY2 controls lifestyle transitions in the fungus Metarhizium robertsii
}

\author{
Na Guo ${ }^{1}$, Ying Qian ${ }^{1,4}$, Qiangqiang Zhang ${ }^{1}$, Xiaoxuan Chen ${ }^{1}$, Guohong Zeng ${ }^{1}$, Xing Zhang ${ }^{1}$, Wubing Mi \\ Chuan $\mathrm{Xu}^{1}$, Raymond J. St. Leger ${ }^{2} \&$ Weiguo Fang ${ }^{1,3}$
}

Metarhizium robertsii is a versatile fungus with saprophytic, plant symbiotic and insect pathogenic lifestyle options. Here we show that $M$. robertsii mediates the saprophyte-toinsect pathogen transition through modulation of the expression of a membrane protein, Mr-OPY2. Abundant Mr-OPY2 protein initiates appressorium formation, a prerequisite for infection, whereas reduced production of Mr-OPY2 elicits saprophytic growth and conidiation. The precise regulation of Mr-OPY2 protein production is achieved via alternative transcription start sites. During saprophytic growth, a single long transcript is produced with small upstream open reading frames in its $5^{\prime}$ untranslated region. Increased production of Mr-OPY2 protein on host cuticle is achieved by expression of a transcript variant lacking a small upstream open reading frame that would otherwise inhibit translation of Mr-OPY2. RNA-seq and qRT-PCR analyses show that Mr-OPY2 is a negative regulator of a transcription factor that we demonstrate is necessary for appressorial formation. These findings provide insights into the mechanisms regulating fungal lifestyle transitions.

\footnotetext{
${ }^{1}$ Institute of Microbiology, Zhejiang University, Hangzhou 310058, China. ${ }^{2}$ Department of Entomology, University of Maryland, College Park, MD 20742 , USA. ${ }^{3}$ Institute of Insect Sciences, Zhejiang University, Hangzhou 310058, China. ${ }^{4}$ Present address: Chongqing Vocational College of Transportation, Chongqing 402247, China. Na Guo and Ying Qian contributed equally to this work. Correspondence and requests for materials should be addressed to W.F. (email: wfang1@zju.edu.cn)
} 
M any fungal pathogens of plants, insects and mammals can switch between parasitic, saprophytic and symbiotic lifestyles in response to changing environmental conditions. Understanding the mechanisms by which they switch between these disparate lifestyles has important implications for agriculture and medicine ${ }^{1-4}$. Metarhizium robertsii is an excellent example of a fungus with multifactorial lifestyles ${ }^{5}$. It is well adapted to life in the soil as a saprophyte, and some isolates such as ARSEF2575 (Mr2575) can colonize plant roots and promote plant growth ${ }^{6-9}$. Mr2575 is also an entomopathogen with an ability to kill a wide spectrum of insects, and accordingly has been developed as a biocontrol agent against agricultural pests and vectors of human diseases ${ }^{10}$.

The transition from saprophyte to pathogen is initiated when conidia adhere to the cuticle of a susceptible insect host and produce germ tubes, which differentiate into infection structures called appressoria (sticky holdfasts that attach to the cuticle). The appressoria produce infection pegs which penetrate the cuticle via a combination of mechanical pressure and cuticle-degrading enzymes. The fungus proliferates in the host hemocoel as a yeastlike phase (blastospores), and the insect is killed by a combination of fungal growth and toxins. Hyphae subsequently re-emerge from the cadaver to produce conidia that have the potential to enter into saprophytic, symbiotic or pathogenic lifestyles ${ }^{11}$.

Many genes involved in the lifestyle switch to pathogen have been experimentally characterized; their gene products are generally expressed at low levels outside the host. They include an adhesin (MAD1) and hydrophobins that are responsible for adherence to the cuticle ${ }^{12,13}$, two chitin synthases for appressorial formation ${ }^{14}$, and a large number of cuticle-degrading enzymes for penetration of the cuticle ${ }^{15,16}$. Gene products associated with colonizing the hemocoel include the cold shock protein CRP1, laccase Mlac1, sterol carrier Mr-NPC2a, the collagen-like protein MCL1, enzymes for anaerobic respiration, and toxic secondary metabolites such as destruxins ${ }^{17-22}$. Major signaling pathway MAPK cascades and cAMP-PKA have been found to regulate both saprophytic growth and pathogenesis ${ }^{23,24}$. Nevertheless, no mechanisms have been characterized that control M. robertsii's choice of lifestyle options as a saprophyte or a pathogen.

Here, from analysis of a random T-DNA insertion library ${ }^{22}$, we identify a membrane anchor protein (Mr-OPY2) that controls the saprophyte-to-pathogen transition of $M$. robertsii. Mr-OPY2 protein levels are low during saprophytic growth, and when elevated they initiate appressorial formation. Precise regulation of the Mr-OPY2 protein level is achieved via alternative transcription start sites. We further find that Mr-OPY2 controls appressorial formation by regulating a previously unidentified transcription factor, AFTF1 (appressorial formation transcription factor 1).

\section{Results}

Identification of the membrane anchor protein Mr-OPY2. From a T-DNA insertion mutant library, we identified a mutant (M2880) that cannot infect insects. Gene mapping showed that the T-DNA in the mutant was inserted in the open reading frame (ORF) of a gene (MAA_03000) that encodes a protein with significant similarity $\left(1 \mathrm{e}^{-08}\right)$ to the membrane anchor protein OPY2p from Saccharomyces cerevisiae (AJV94457), and we designated this gene as $M r-O P Y 2$ ( $M$. robertsii OPY2). Mr-OPY2 is a single copy gene with an 1,305 bp ORF that is interrupted by one intron, and encodes a protein containing 434 amino-acid residues. The deduced Mr-OPY2 has an OPY2 domain (PFAM09463) from amino acids 30 to 68, and a transmembrane domain from amino acids $93-115$ as predicted by TMHMM (version 2.0) ${ }^{25}$, and domains showing significant similarity to CR$\mathrm{A}$ and $\mathrm{D}$ in $S$. cerevisiae Opy2 $\mathrm{p}^{26}$. Blast analysis found that homologs of Mr-OPY2 were widely distributed in both

Table 1 Transcripts and strains in this study

Name

\section{Transcripts}

Mr-OPY2-S

$5^{\prime} U T R^{S}$

Mr-OPY2-L

5' UTR ${ }^{L}$

Mr-OPY2-L $\triangle A \cup G s$

Genomic clones

gMr-OPY2

gMr-OPY2 ${ }^{\triangle A U G s}$

Strains

WT

M2880

$\triangle \mathrm{Mr}-\mathrm{OPY} 2$

$\mathrm{C}-\triangle \mathrm{Mr}-\mathrm{OPY} 2$

$\triangle M r-S T E 50$

C- $\triangle M r-S T E 50$

T-Mr-OPY2-LA AUGs

T-Mr-OPY2-LAUG

$\triangle M r-O P Y 2: M r-O P Y 2-S$

$\triangle M r-O P Y 2: M r-O P Y 2-L$

$\triangle M r-O P Y 2:: M r-O P Y 2-L \Delta A U G s$

P404-Mr-OPY2

P683-Mr-OPY2

$\Delta$ Mero-Fus3

$\Delta$ Mero-Hog1

$\Delta$ Aftf1

$C-\Delta$ Aftf1

Aftf1 $O E$

\section{Description}

The short transcript of the Mr-OPY2 gene

The 5'UTR of the short transcript Mr-OPY2-S

The long transcript of the Mr-OPY2 gene

The 5'UTR of the long transcript Mr-OPY2-L

The mutant of Mr-OPY2-Lwith the 5'UTR ${ }^{\mathrm{L}}$ uORFs mutated

The genomic clone of the Mr-OPY2 gene

The mutant of gMr-OPY2 with the 5'UTR uORFs mutated

The wild-type strain of $M$. robertsii ARSEF2575

A mutant with T-DNA inserted in the ORF of the Mr-OPY2 gene

The mutant with Mr-OPY2 ORF deleted based on homologous recombination

The complemented strain of $\triangle M r-O P Y 2$

The mutant with Mr-STE50 ORF deleted based on homologous recombination

The complemented strain of $\triangle M r$-STE50

The strain with $g M r-O P Y 2 \Delta^{A U G s}$ to replace its corresponding region in $M$. robertsii

The strain with $g M r-O P Y 2$ to replace its corresponding region in $M$. robertsii

The strain with Mr-OPY2-S controlled by the constitutive promoter Pgpd

The strain with Mr-OPY2-L controlled by the promoter Pgpd from M. acridum

The strain witht Mr-OPY2-LA AUGs controlled by the Pgpd from M. acridum

The strain with Mr-OPY2 mORF driven by the 404 bp Pgpd from M. acridum

The strain with Mr-OPY2 mORF driven by the 683 bp Pgpd from M. acridum

The deletion mutant of the Fus3-MAPK gene

The deletion mutant of the Hog1-MAPK gene

The deletion mutant of the Aftf1 gene

The complemented strain of $\Delta A f t f 1$

The strain overexpressing the Aftf1 gene
Ref

This study

This study

This study

This study

This study

This study

This study

This study

This study

This study

This study

This study

This study

This study

This study

This study

This study

Ref. 24

Ref. 24

This study

This study

This study 
a

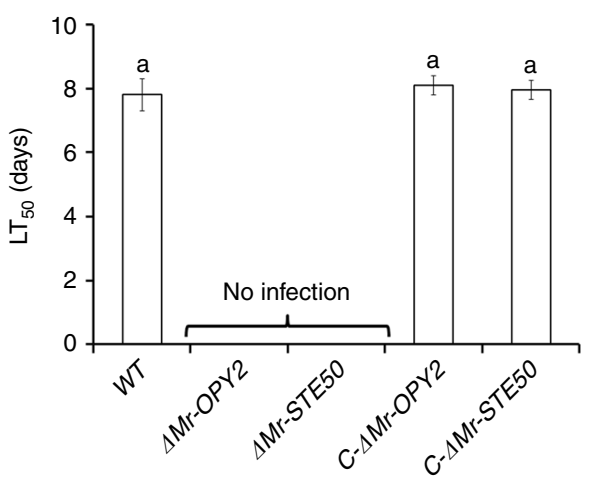

b

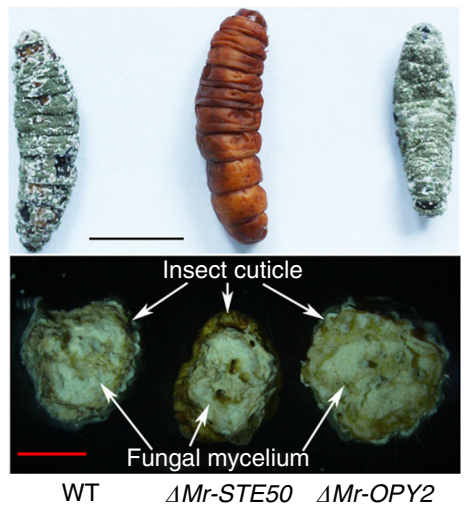

C
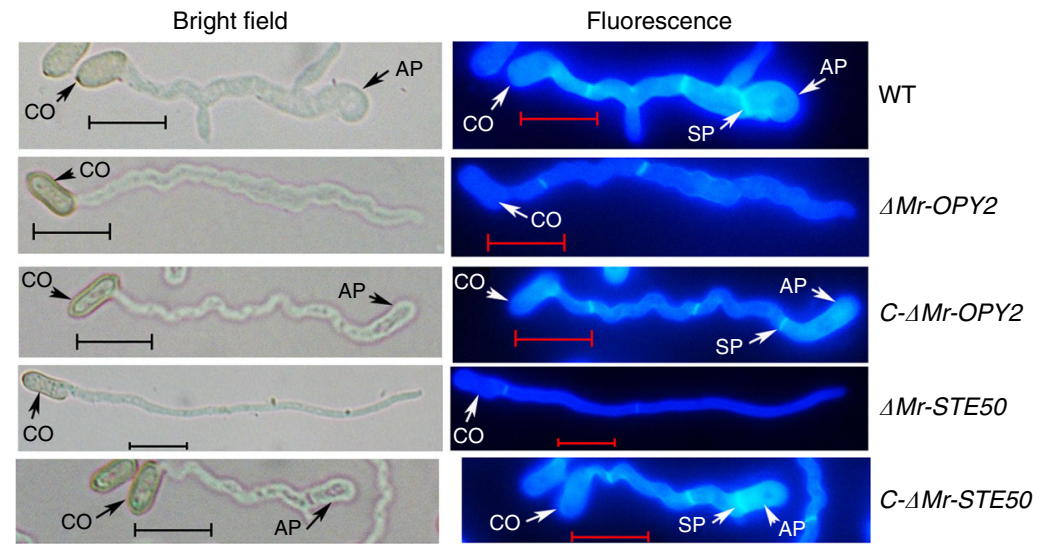

Fig. 1 Pathogenicity of $\mathrm{WT}$, the mutants $\triangle M r-O P Y 2$ and $\triangle M r-S T E 50$, and their respective complementation strains. a $L_{50}$ (time taken to kill $50 \%$ of insects) values when the insects were inoculated by topical application. The bioassays were repeated three times with 40 insects per repeat. Data are expressed as the mean \pm SE. Values with different letters are significantly different $(n=3, P<0.05$, Tukey's test in one-way ANOVA). b Upper panel: mycelial growth and conidiation on the surface of cadavers (scale bar represents $10 \mathrm{~mm}$ ). Lower panel: mycelia in cross sections of cadavers (scale bar represents $5 \mathrm{~mm}$ ). Each image is representative of $\sim 120$ insect cadavers (three replicates with 40 insects per replicate). c Formation of appressoria (stained with Calcofluor Brightener White 2B) against a hydrophobic plastic surface. AP: appressorium; CO: conidium; SP: septum. Left: bright field microscopy. Note: the hyphal tips of $\triangle M r-O P Y 2$ and $\triangle M r-S T E 50$ do not swell to form appressoria. Right: fluorescence microscopy. Note: the septum between the appressorium and its appressorial mother cell. Images are representative of at least three independent experiments for each condition. Scale bar represents $10 \mu \mathrm{m}$

pathogenic and saprophytic Ascomycota and Basidiomycota. Phylogenetic analysis showed that the clade containing OPY2 proteins from Ascomycota yeasts was basal to the clade that contained OPY2 proteins of filamentous Ascomycota and Basidiomycota. The OPY2 genes of the filamentous fungi conform to their species tree, consistent with their being derived from a common ancestral sequence (Supplementary Fig. 1a, Supplementary Table 1). We compared the topology of the obtained tree with those of alternative trees using nine tests including the $\mathrm{SH}$-test and the $\mathrm{AU}$-test provided by the program CONSEL $^{27}$. The nine tests consistently showed that the obtained tree (Supplementary Fig. 1a) to be the best supported (Supplementary Fig. 1b).

Mr-OPY2 and pathogenicity. We disrupted $M r-O P Y 2$ in the wild-type strain (WT) to produce $\triangle M r$-OPY2. The genes and strains used in this study are listed in Table 1. $\Delta M r-O P Y 2$ was complemented with its genomic clone containing the upstream $(1,747 \mathrm{bp})$ and downstream (245 bp) regions to produce strain $C$ $\triangle M r-O P Y 2$. The confirmation of gene disruption and mutant complementation is presented in Supplementary Fig. 2.

The pathogenicity of $M$. robertsii was assayed on Galleria mellonella larvae. Inoculations were conducted either by topically applying conidia onto the insect cuticle or by direct injection of conidia into the hemocoel (thus bypassing the cuticle). As with the T-DNA insertion mutant M2880, $\triangle M r$-OPY2 was unable to infect insects via topical application (Fig. 1a), but it was as pathogenic as the WT following injection (Supplementary Fig. 3). $C-\Delta M r-O P Y 2$ showed WT levels of virulence (Supplementary Fig. 3) following topical application or direct injection (Fig. 1a and Supplementary Fig. 3). Insects killed by either the WT or injected $\triangle M r$-OPY2 were mummified with dark green conidia produced on the cadavers (Fig. 1b).

We investigated whether the mutants were able to produce appressoria against insect cuticle and another normally inductive milieu-the hydrophobic surface of plastic petri dishes in the presence of low levels of nitrogenous nutrients ${ }^{28} . \Delta M r-O P Y 2$ germ tubes meandered across the plastic and hindwings of Locusta migratoria manilensis, but their hyphal tips did not swell to initiate appressorial formation. Fluorescent staining with Calcofluor white Brightener 2B showed that the fluorescent intensity of the $\triangle M r-O P Y 2$ hyphal tip was not distinguishable from other parts of the hyphae (Fig. 1c), whereas both WT and $C-\triangle M r-O P Y 2$ produce normal appressoria that fluoresce strongly (Fig. 1c). Therefore, the cell wall structure or composition is altered in the growing hyphal tips of $\triangle M r-O P Y 2$. In addition, appressorial differentiation of $M$. robertsii typically occurs after nuclear division with a septum being formed between the 

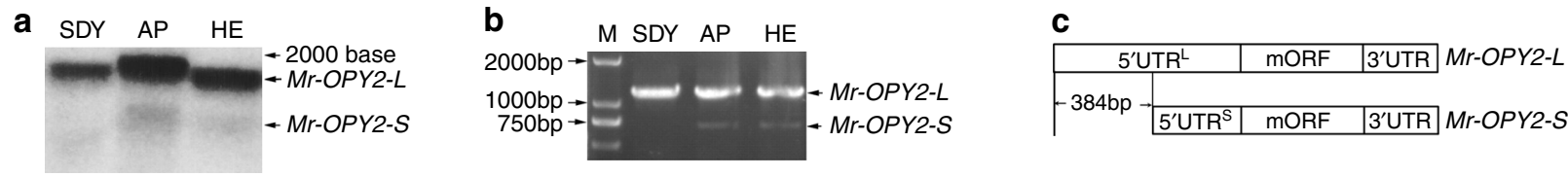

d

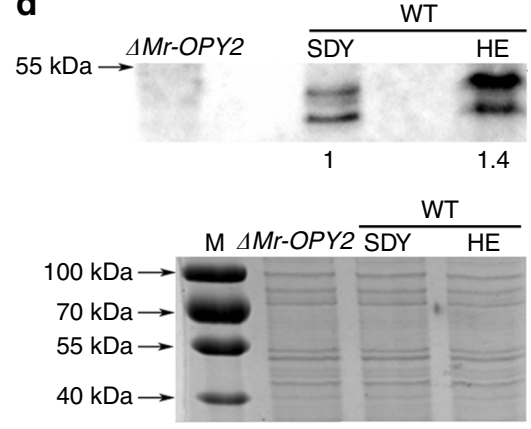

e
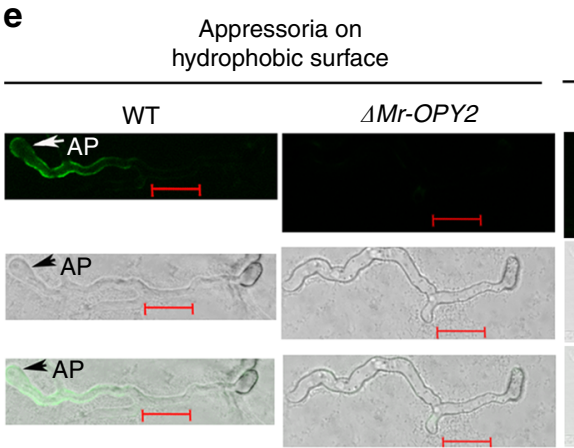
growth in $1 / 2$ SDY

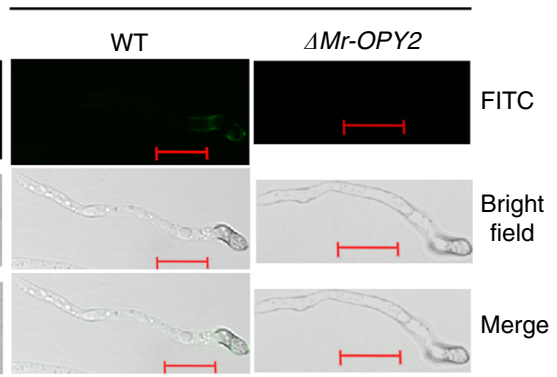

Fig. 2 Transcription and translation analyses of the Mr-OPY2 gene. a Representative Northern blot image of RNA isolated from undifferentiated mycelia grown in SDY or hemolymph (HE), and from germlings differentiating appressoria on locust wings (AP). The uncropped Northern blot is shown in Supplementary Fig. 11a. b 5'RACE with the RLM RT-PCR kit. The uncropped image of the agarose gel is shown in Supplementary Fig. 11b. c A diagrammatic representation of differences between the two mRNA variants of Mr-OPY2. Note: the two transcripts share the same major ORF but they have different $5^{\prime}$ UTR. d Representative western blot image and analysis to quantify Mr-OPY2 levels in $\triangle M r-O P Y 2$ and WT grown in SDY or hemolymph (HE) (Upper panel). The OPY2 levels of hemolymph-grown WT mycelium (1.4) is calculated relative to growth in SDY which is set at 1. $\triangle M r-O P Y 2$ is included as a negative control. Lower panel: a portion of the loading control gel (for the Western blot analysis in upper panel) stained with Coomassie Brilliant Blue. M: Protein ladder (Thermo scientific, USA). The uncropped images of the western blot and the SDS-PAGE gel are shown in Supplementary Fig. 11c, d. e Immunohistochemical staining of the Mr-OPY2 protein in germlings differentiating appressoria against a plastic surface, and in non-differentiating germlings from $1 / 2$ SDY shake cultures. Scale bar represents $10 \mu \mathrm{m}$. FITC: Fluorescein isothiocyanate; Merge: FITC and bright field pictures are merged; AP: appressorium. Note: no fluorescent signal in $\triangle M r-O P Y$ and a weak signal in WT grown in 1/2 SDY. Images are representative of at least three independent experiments for each condition

appressorium and appressorial mother cell ${ }^{28}$. This septation was observed in WT and $C-\triangle M r-O P Y 2$, but not in $\triangle M r-O P Y 2$ (Fig. 1c). WT and $C-\Delta M r-O P Y 2$ germlings typically had three septa when the hyphal tips initiated appressorial formation, at which time $\triangle M r$-OPY2 only had two. $18 \mathrm{~h}$ after inoculation, when $\sim 80 \%$ of WT hyphal tips had produced appressoria against plastic surfaces, the hyphae of WT $(27.4 \pm 1.82 \mu \mathrm{m})$ were significantly ( $t$-test, $P=0.007)$ shorter than $\triangle M r$-OPY2 $(42.7 \pm$ $0.29 \mu \mathrm{m})$, as $\Delta M r$-OPY2 showed continued polar growth rather than differentiation into appressoria.

Mr-OPY2 has two mRNA variants during infection. Northern blots and $5^{\prime}$ and $3^{\prime}$ RACE (Rapid Amplification of cDNA Ends) were used to analyze RNA during: (1) saprophytic growth (in SDY broth (Sabouraud dextrose broth plus 1\% yeast extract)); (2) cuticle penetration (conidia germinating and differentiating appressoria on locust wings) and 3) hemocoel colonization (hyphae cultured in Bombyx mori (silkworm) hemolymph). Northern analysis revealed that a single $M r$-OPY2 transcript was produced during saprophytic growth, and an additional less intense smaller band was produced in the two infection stages (Fig. 2a). RACE was performed using the RLM RT-PCR kit (Roche, USA) that only clones intact mRNA with a 7-methyl guanosine cap structure. Only one PCR band was obtained from the three RNA samples with $3^{\prime}$ RACE. Consistent with Northern analysis, $5^{\prime}$ RACE obtained one long band using RNA from $M$. robertsii mycelia grown in SDY broth, and an additional shorter band from $M$. robertsii when penetrating cuticle or growing in hemolymph (Fig. 2b). All PCR products were cloned, sequenced and assembled. The long $1,836 \mathrm{bp}$ and shorter $1,452 \mathrm{bp}$ transcripts are designated as Mr-OPY2-L (Genbank number: KY548479), and Mr-OPY2-S (Genbank number: KY548480), respectively. The two mRNA variants contain an identical ORF (designated as major ORF:mORF) that encodes Mr-OPY2 (Fig. 2c), and differ only in that the $5^{\prime}$ UTR of $M r-O P Y 2-L$ (designated as $5^{\prime} \mathrm{UTR}^{\mathrm{L}}$ ) is $384 \mathrm{bp}$ longer than that of $\mathrm{Mr}-O P Y 2-\mathrm{S}$ (designated as $5^{\prime} \mathrm{UTR}^{S}$ ). The first nucleotide of $M r-O P Y 2-L(\mathrm{~T})$ is different from that of $M r-O P Y 2-S(C)$, and no intron was found in the region corresponding to the UTRs, indicating that the two mRNA variants result from alternative transcription start sites.

Western blot analysis showed that there was 1.4-fold more Mr-OPY2 protein in hemolymph cultures than in SDY (Fig. 2d). We could not obtain sufficient biomass from differentiating appressoria to extract membrane proteins for western analysis, so we used indirect immunofluoresence (IIF) assays to compare Mr-OPY2 protein levels in appressoria with those in nondifferentiating germlings in SDY. The assay results showed that the Mr-OPY2 protein was more abundant in appressoria (Fig. 2e).

To identify differences in copy number between $M r$-OPY2-L and $M r-O P Y 2-S$ we used two pairs of primers for quantitative RT-PCR (qRT-PCR) analysis of the $5^{\prime} \mathrm{UTR}^{\mathrm{L}}$ and mORF (Supplementary Fig. 4). No significant differences were found in the levels of $5^{\prime} \mathrm{UTR}^{\mathrm{L}}$ and mORF during saprophytic growth and the two infection stages. Therefore, expression of the Mr-OPY2-S transcript did not significantly contribute to mORF copy number. Consequently, changes in Mr-OPY2 protein levels during lifestyle transitions are most probably attributable to translational regulation of mORF in $M r-O P Y 2-L$ and $M r-O P Y 2-S$.

uORFs mediate translational suppression of mORF in $\mathrm{Mr}$ OPY2-L. The $5^{\prime} \mathrm{UTR}^{\mathrm{S}}$ of $M r$-OPY2-S lacks uORFs (upstream ORFs), whereas two uORFs were identified in the $5^{\prime} \mathrm{UTR}^{\mathrm{L}}$ of $M r-O P Y 2-L$ (Fig. 3a). As uORFs suppress translation efficiency in many genes ${ }^{29-31}$, we investigated the effect of the uORFs on translation of the main ORF in Mr-OPY2-L. To do this, we cloned 
a

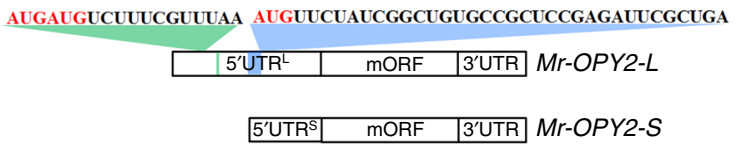

GUGGUGUCUUUCGUUUA GUGUUCUAUCGgCUGUGCCGCUCCGagaUUCGCUGA

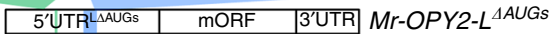

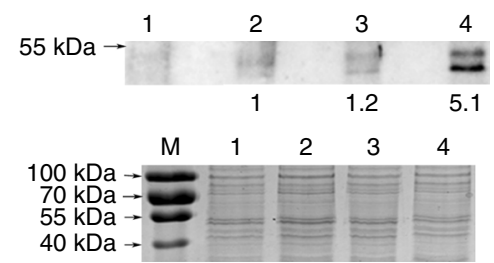

b
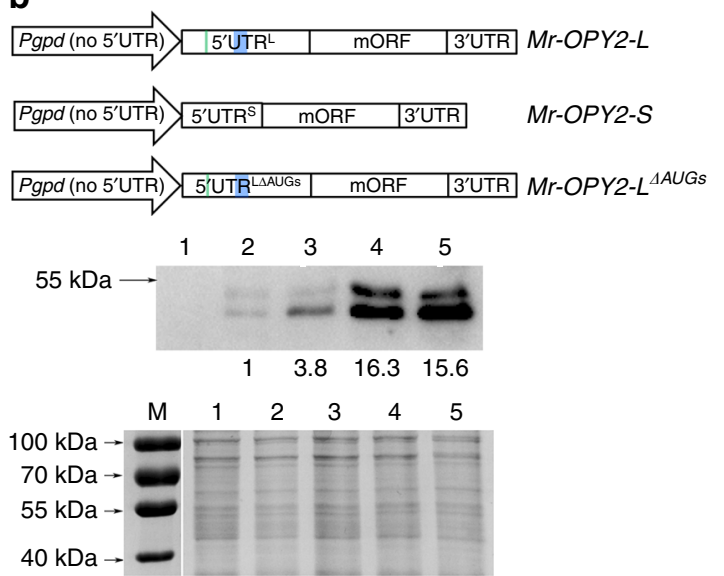

Fig. 3 The uORFs suppress translation efficiency of the major ORF in Mr-OPY2-L. a Top panel: graphic representation of WT transcripts Mr-OPY2-L, MrOPY2-S and the mutated Mr-OPY2-L (Mr-OPY2-LAUGs) that has GUGs in place of AUGs (highlighted in red). The blue and green labelled sequences in the $5^{\prime}$ UTR represent the positions of the two different uORFs (these color conventions are also used in the top panel in $\mathbf{b}$. Middle panel: Mr-OPY2 protein levels in SDY grown cultures of: (1) $\triangle M r-O P Y 2 ;(2) W T ;$ (3) T-Mr-OPY2- $L^{A U G}$ (the native Mr-OPY2 gene was replaced by a native genomic Mr-OPY2 clone); and (4) T-Mr-OPY2 ${ }^{\triangle A U G s}$ (the native Mr-OPY2 gene was replaced by a genomic Mr-OPY2 clone with mutated uORFs). Bottom panel: a portion of the loading control gel (for the Western blot analysis in the middle panel) stained with Coomassie Brilliant Blue. M: Protein ladder. b The short Mr-OPY2-S transcript is more efficiently translated than the longer Mr-OPY2-L. Top panel: graphic representation of the constructs containing the transcript Mr-OPY2L, Mr-OPY2-S, and Mr-OPY2-L ${ }^{\triangle A U G s}$ driven by the Pgpd-NUTR. The 'No 5'UTR' indicates that the 5'UTR of the M. acridum gpd gene is excluded. These constructs were transformed into the Mr-OPY2 deletion mutant $\triangle M r-O P Y 2$. Middle panel: representative Western blot image and analysis to quantify MrOPY2 levels in SDY grown cultures of: (1) $\triangle M r-O P Y 2$; (2) WT; (3) $\triangle M r-O P Y 2:: M r-O P Y 2-L ;$ (4) $\triangle M r-O P Y 2:: M r-O P Y 2-S$, and (5) $\triangle M r-O P Y 2:: M r-O P Y 2-L A U G s$. Bottom panel: a portion of the loading control gel (for the western blot analysis in the middle panel) stained with Coomassie Brilliant Blue. M: Protein ladder. For western blot analysis ( $\mathbf{a}, \mathbf{b}$ middle panels), the Mr-OPY2 levels are calculated relative to the WT which is set at 1 ( $\triangle M r-O P Y 2$ is a negative control). The uncropped images of the western blots and the SDS-PAGE gels are shown in Supplementary Fig. 12. Images are representative of at least three independent experiments

the genomic DNA fragment ( $g M r-O P Y 2)$ that contains the two exons, the single intron and the termination region $(200 \mathrm{bp})$, and mutated the AUGs to GUGs in the $5^{\prime} \mathrm{UTR}^{\mathrm{L}}$ to generate $g M r$ $O P Y 2^{\triangle A U G s}$ (Fig. 3a, Supplementary Fig. 5a). The knock-in method was used to replace the corresponding region in $M$. robertsii's genome with $g M r-O P Y 2^{\triangle A U G s}$, producing the strain $T$ $M r-O P Y 2-L^{\triangle A U G s}$. As a control, the corresponding region was also replaced with the unmutated $g M r-O P Y 2$ to produce the strain T-Mr-OPY2 $2^{A U G}$ (Supplementary Fig. 5a, b). qRT-PCR of SDY mycelial cultures revealed that expression of the Mr-OPY2 ORF was similar $(P>0.05$, Tukey's test in one-way analysis of variance (ANOVA)) in WT, T-Mr-OPY2 ${ }^{A U G}$ and $T-M r-$ $O P Y 2^{\triangle A U G s}$ (Supplementary Fig. 5c), whereas western analysis showed that Mr-OPY2 protein levels in $T-M r-O P Y 2^{\triangle A U G s}$ were increased $\sim 4$-fold compared with the WT and T-Mr-OPY2AUG (Fig. 3a). No obvious difference in the level of Mr-OPY2 protein was observed between WT and T-Mr-OPY2-L ${ }^{A U G}$ (Fig. 3a). These results show that upstream AUG triplets in the $5^{\prime}$ UTR of the long mRNA variant of Mr-OPY2 reduce translatability and protein levels.

Secondary structures of $5^{\prime}$ UTRs may affect translation of the downstream ORF. We thus predicted the secondary structures of $5^{\prime} \mathrm{UTR}^{\mathrm{L}}$ and $5^{\prime} \mathrm{UTR}^{\mathrm{S}}$ using the Sfold Web Server (http://sfold. wadsworth.org/cgi-bin/index.pl). The $5^{\prime} \mathrm{UTR}^{\mathrm{L}}$ formed a complex secondary structure with many stem-loops, and had a Gibbs free energy $\Delta \mathrm{G}_{37}^{0}=-25.6 \mathrm{Kcal} \mathrm{mol}^{-1}$. In contrast, $5^{\prime} \mathrm{UTR}^{\mathrm{S}}$ formed a simple stem-loop structure with $\Delta \mathrm{G}^{0}{ }_{37}=-7.2 \mathrm{Kcal} \mathrm{mol}^{-1}$. The secondary structure of the 5' UTR of Mr-OPY2-L $L^{\Delta A U G s}\left(\Delta \mathrm{G}_{37}^{0}=\right.$ $-26.9 \mathrm{Kcal} / \mathrm{mol}$ ) was similar to that of $M r$-OPY2-L (Supplementary Fig. 5d). Secondary structures with free energy higher than $\Delta \mathrm{G}=-30 \mathrm{Kcal} / \mathrm{mol}$ are not considered to impair translation ${ }^{32}$. Therefore, it is unlikely that the secondary structures of $5^{\prime} \mathrm{UTR}^{\mathrm{L}}$ and $5^{\prime} \mathrm{UTR}^{\mathrm{S}}$ substantially effect translation of the downstream ORF.
To characterize the effects of $5^{\prime} \mathrm{UTR}^{\mathrm{L}}$ and $5^{\prime} \mathrm{UTR}^{\mathrm{S}}$ further, we constructed expression plasmids where the transcripts $\mathrm{Mr}$-OPY2$S, M r-O P Y 2-L$ and $M r-O P Y 2-L^{\triangle A U G s}$ were placed downstream of the constitutive $g p d$ gene promoter $(P g p d)$ from M. acridum ${ }^{33}$. The $5^{\prime}$ UTR of the gpd gene was not included in the promoter to avoid it impacting translation (Fig. 3b). These plasmids were then transferred into $\triangle M r-O P Y 2$ (i.e., the native mORF was deleted) to construct strains $\triangle M r-O P Y 2:: M r-O P Y 2-S, \quad \Delta M r-O P Y 2:: M r-$ $O P Y 2-L$, and $\triangle M r-O P Y 2:: M r-O P Y 2-L^{\triangle A U G s}$. Strains with similar levels of the mORF transcript were selected for further analysis (Supplementary Fig. 6). In SDY cultures, the level of Mr-OPY2 protein in $\triangle M r-O P Y 2:: M r-O P Y 2-S$ was $\sim 4$-fold higher than $\Delta M r$ $O P Y 2: M r-O P Y 2-L$, indicating that $M r-O P Y 2-S$ was more efficiently translated than $M r-O P Y 2-L$. Conversely, $\triangle M r-O P Y 2:$ : $M r-O P Y 2-L^{\triangle A U G s}$ and $\triangle M r-O P Y 2: M r-O P Y 2-S$ had similar MrOPY2 protein levels (Fig. 3b). Taken together these results suggest that: (1) the $5^{\prime} \mathrm{UTR}^{\mathrm{L}}$ secondary structure does not affect the translation efficiency of mORF, and (2) uORFs are major factors for suppressing the translation of mORF.

Differences in Mr-OPY2 levels lead to lifestyle transitions. As described above, alternative transcription start sites produce differences in Mr-OPY2 protein levels that characterize saprophytic and pathogenic lifestyles. In order to investigate the impact of Mr-OPY2 protein abundance on lifestyle transitions, we took advantage of a series of truncated Pgpd promoters of the $M$. acridum gpd gene which express foreign genes at different strengths ${ }^{33}$. We selected the $404 \mathrm{bp}$ (P404) and $683 \mathrm{bp}$ (P683) long promoters, which include the $5^{\prime} \mathrm{UTR}$ of the $M$. acridum gpd gene, to drive the major ORF of $M r-O P Y 2$ in the $M r-O P Y 2$ deletion mutant ( $\triangle M r-O P Y 2)$ (Fig. 4 a), i.e., the expression of $M r-$ OPY2 was exclusively controlled by the constitutive gpd promoters. As expected, qRT-PCR analysis showed that expression 

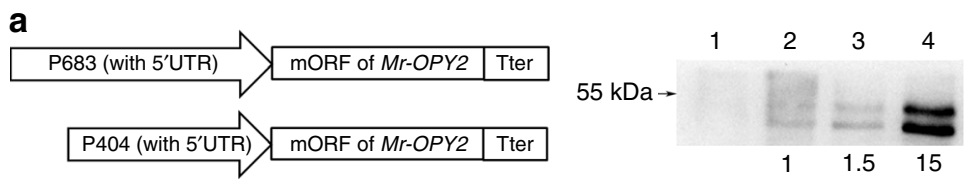

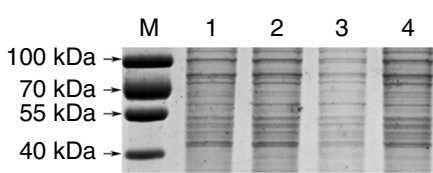

b
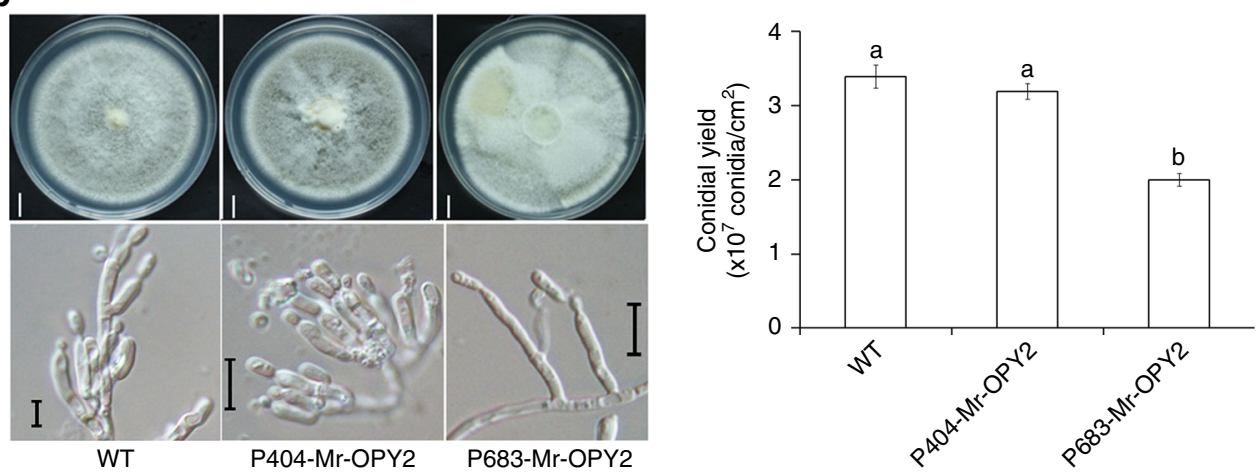

C
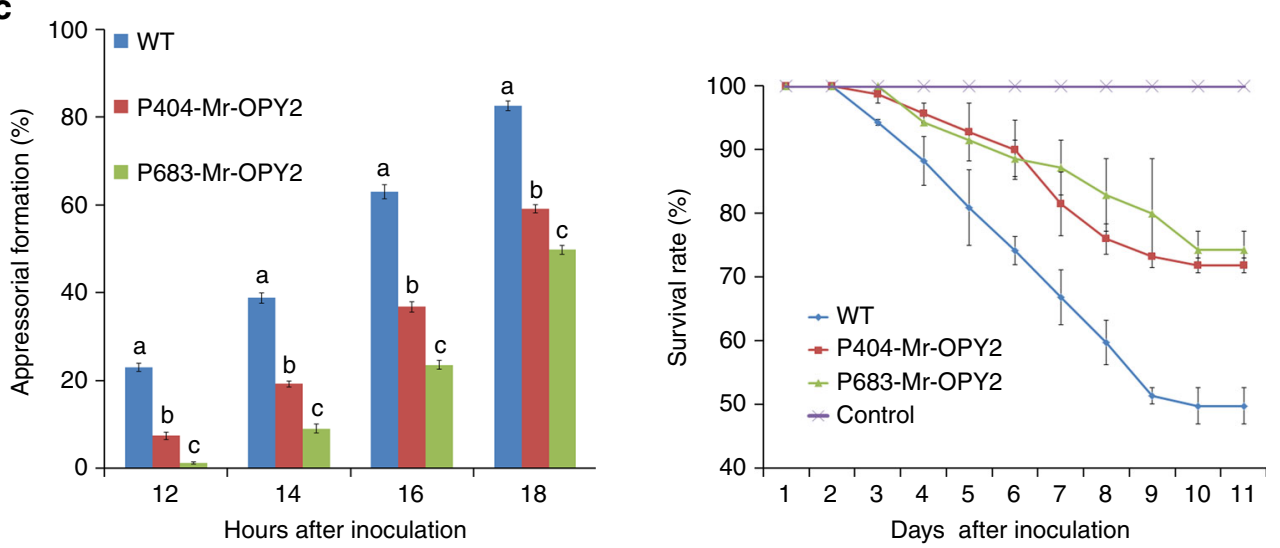

Fig. 4 Precise regulation of Mr-OPY2 levels is important for saprophytic growth and infection. a Constructing strains with manipulated Mr-OPY2 levels. Left panel: diagram illustrating constructs where Mr-OPY2's ORF is driven by the two Pgpd promoters, P683 and P404, with high and low activity, respectively. These constructs were transformed into $\triangle M r-O P Y 2$ to produce strains where the Mr-OPY2 level was exclusively controlled by the promoters. The 'With 5'UTR' indicates the 5'UTR of the M. acridum gpd gene is included. Middle panel: western blot analysis of Mr-OPY2 levels in the transformants: (1) $\triangle$ Mr-OPY2; (2) WT; (3) P404-Mr-OPY2; and (4) P683-Mr-OPY2. Mr-OPY2 levels are calculated relative to the WT which is set at 1. $\Delta$ Mr-OPY2 is a negative control. Right panel: a portion of the loading control gel (for the Western blot analysis in the middle panel) stained with Coomassie Brilliant Blue. M: Protein ladder. The uncropped images of the western blot and the SDS-PAGE gel are shown in Supplementary Fig. 13. b Colony phenotype (left upper panel), conidiophores (left lower panel) and conidial yields (right panel) of strains with different Mr-OPY2 levels. Note: strain P683-Mr-OPY2 with elevated Mr-OPY2 level relative to WT has a fluffy colony with impaired conidiophores and reduced conidial yield. Colony pictures were taken 18 days after inoculation. Scale bar in the left upper panel represents $10 \mathrm{~mm}$, left lower panel $10 \mu \mathrm{m}$. In the right panel, values with different letters are significantly different $(n=9, P<0.05$, Tukey's test in one-way ANOVA). c Pathogenicity of strains with different Mr-OPY2 levels. Left panel: appressorial development on a hydrophobic surface. At each time point, values with different letters are significantly different $(n=3, P<0.05$, Tukey's test in one-way ANOVA). Right panel: survival curves of G. mellonella larvae infected by the WT, P404-Mr-OPY2 and P683-Mr-OPY2. Control: insects treated with 0.01\% Triton X-100 solution. Bioassays were repeated three times with 40 insects per repeat. Conidial yield and appressorium formation assays were repeated three times with three replicates per repeat. Data are expressed as the mean \pm SE. Images are representative of at least three independent experiments for each condition

of the Mr-OPY2 transcript in P683-Mr-OPY2 (driven by the promoter P683) is significantly higher $(t$-test, $P=0.006)$ than expression driven by P404 (strain designated P404-Mr-OPY2), and the quantity of Mr-OPY2 protein in P683-Mr-OPY2 was $\sim 8$ fold higher than in either P404-Mr-OPY2 or WT (Fig. 4a). Three isolates were randomly selected from $P 404-M r-O P Y 2$ and $P 683-$ $\mathrm{Mr}$-OPY2 colonies. As no intra-strain differences were found between the three isolates of each strain in any assay we provide representative data for a single isolate/strain.

During saprophytic growth on PDA (potato dextrose agar) plates, the growth rate and conidial yield of P404-Mr-OPY2 was similar to WT (Fig. 4b). However, the more highly expressing
P683-Mr-OPY2 produced a fluffy phenotype, abnormal conidiophores and significantly $(P<0.01$, Tukey's test in one-way ANOVA) lower conidial yield than WT (Fig. 4b). Compared with WT, appressorial formation by P404-Mr-OPY2 and P683$M r-O P Y 2$ was delayed (Fig. 4c), and the pathogenicity of both P683-Mr-OPY2 and P404-Mr-OPY2 was significantly $(P<0.05$, Tukey's test in one-way ANOVA) reduced (Fig. 4c).

Mr-OPY2 and tolerance to abiotic stresses. As S. cerevisiae Opy2p is involved in tolerance to high osmotic stress (Yamamoto et al., 2016), we investigated whether M. robertsii OPY2 is also 
a

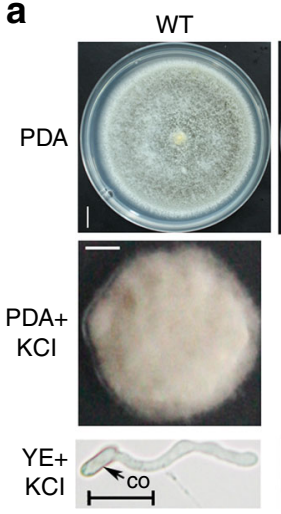

b

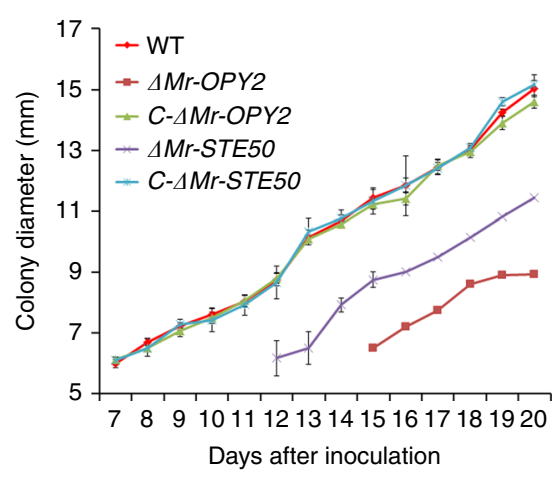

$\triangle M r-S T E 50$

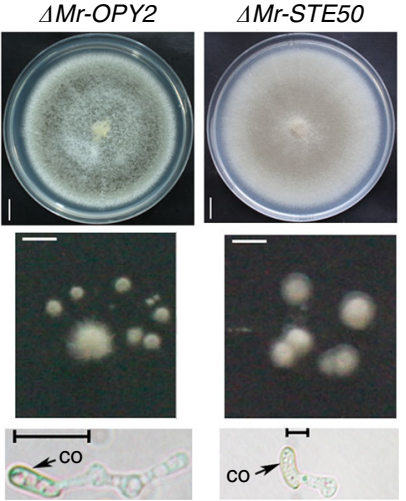

C
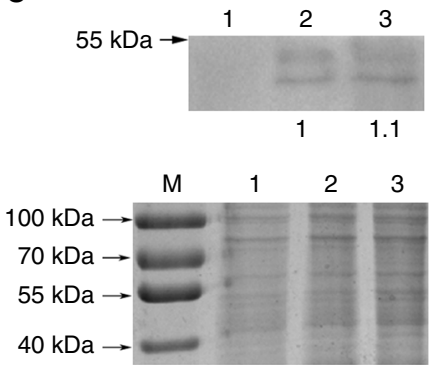

d

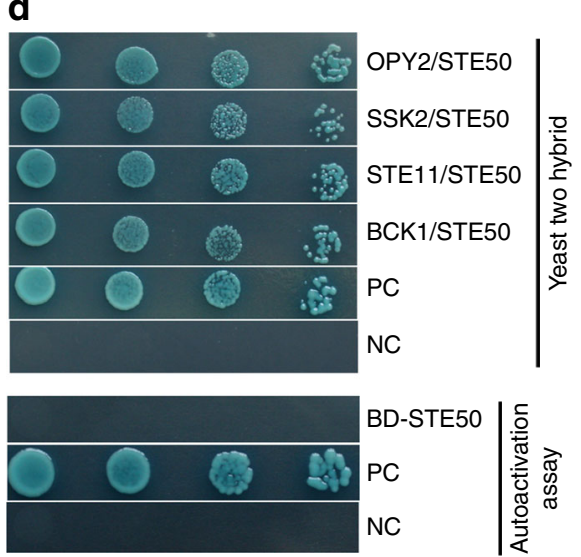

Fig. 5 Colony phenotypes and osmotic stress tolerance of $\triangle M r-O P Y 2, \triangle M r-S T E 50$ and WT. a Upper panel: colonies of the strains on PDA (Scale bar represents $10 \mathrm{~mm}$ ). Middle panel: colonies on PDA plus $0.75 \mathrm{M} \mathrm{KCl}$ (scale bar represents $1 \mathrm{~mm}$ ). Lower panel: bright field images of individual germlings in YE $(0.01 \%$ yeast extract) plus $0.75 \mathrm{M} \mathrm{KCl}$ (Scale bar represents $10 \mu \mathrm{m})$. Colony pictures were taken 18 days after inoculation. Germling pictures were taken $16 \mathrm{~h}$ after inoculation. $\mathbf{b}$ Growth curves of the strains on PDA plates plus $0.75 \mathrm{M} \mathrm{KCl}$. Growth assays were repeated three times with three replicates per repeat. Data are expressed as the mean \pm SE. Note: growth of $\triangle M r-O P Y 2$ and $\triangle M r-S T E 50$ was greatly reduced. c Upper panel: Western blot analysis of Mr-OPY2 protein levels in $\triangle M r-O P Y 2$ grown in SDY (1), WT grown in SDY (2) and in SDY plus $0.75 \mathrm{M} \mathrm{KCl} \mathrm{(3).} \mathrm{The} \mathrm{band} \mathrm{intensity} \mathrm{of} \mathrm{WT} \mathrm{in} \mathrm{SDY} \mathrm{is} \mathrm{set} \mathrm{at} 1$, and $\mathrm{WT}$ in SDY with $\mathrm{KCl}$ is relative to it; $\triangle \mathrm{Mr}-\mathrm{OPY} 2$ is a negative control. Lower panel: a portion of the loading control gel (for the western blot analysis in the upper panel) stained with Coomassie Brilliant Blue. M: Protein ladder. The uncropped images of the Western blot and the SDS-PAGE gel are shown in Supplementary Fig. 13. d Upper panel: yeast two-hybrid confirmation of the physical interaction of Mr-SET50 with Mr-OPY2 and three MAPKKK proteins (SSK2, STE11 and BCK1). Colonies were grown in SD-His-Ade-Leu-Trp + X- $\alpha$-gal + AbA (Takara, Dalian China). NC: negative control (yeast cells containing the plasmid pGADT7-T and pGBKT7-Lam). PC: positive control (yeast cells containing the plasmid pGADT7-T and pGBKT7-53). Lower panel: Mr-STE50 lacks autoactivation activity. Y2HGold cells with pGBKT7-Mr-STE50 cannot grow in SD-His-Trp-Ade + X- $\alpha$-gal (Takara, Dalian China). NC and PC are the same as those in the upper panel. Images are representative of at least three independent experiments for each condition

involved in tolerance to abiotic stresses. $\triangle M r$-OPY2 germinates and grows in SDY broth or on PDA plates at the same rate as WT and $C-\Delta M r-O P Y 2$ (Fig. 5a, Supplementary Fig. 7), and this was not altered by adding $0.01 \% \mathrm{H}_{2} \mathrm{O}_{2}$ to induce oxidative stress (Supplementary Fig. 7). The cell wall-disturbing agent Congo red $\left(1 \mathrm{mg} \mathrm{ml}^{-1}\right)$ produced no significant $(P>0.05$, Tukey's test in One-way ANOVA) differences in growth rate between WT, $\Delta M r$ $O P Y 2$ and $C-\Delta M r-O P Y 2$ on PDA (Supplementary Fig. 7), but the germination rate of $\triangle M r-O P Y 2$ in SDY was significantly reduced $(P<0.05$, Tukey's test in one-way ANOVA) (Supplementary Fig. 7). Under hyperosmotic stress (SDY plus $0.75 \mathrm{M} \mathrm{KCl}$ ), WT, $\triangle M r-O P Y 2$ and $C-\Delta M r-O P Y 2$ showed similar $(P>0.05$, Tukey's test in One-way ANOVA) germination rates, but the $\triangle M r-O P Y 2$ hyphae were severely deformed forming a pearl-like shape (Fig. 5a). Similarly, unlike the $C-\triangle M r$-OPY2 and WT, mycelial growth of $\triangle M r-O P Y 2$ on PDA plates supplemented with $0.75 \mathrm{M}$ $\mathrm{KCl}$ was also greatly $(P<0.01$, Tukey's test in One-way ANOVA) inhibited (Fig. 5b). However, the level of Mr-OPY2 protein in WT was the same in SDY and in SDY supplemented with $0.75 \mathrm{M} \mathrm{KCl}$ (Fig. 5c). No significant differences were observed between WT,
P683-Mr-OPY2 and P404-Mr-OPY2 in their tolerance to oxidative stress, high osmotic stress and Congo red (Supplementary Fig. 8).

Mr-OPY2 controls phosphorylation levels of MAPKs. The $S$. cerevisiae OPY2 protein regulates the phosphorylation level of MAPKs by complexing with the adaptor protein STE50p that in turn interacts with three MAPKKK proteins ${ }^{26}$. We used yeast two-hybrid assays to confirm that the $M$. robertsii Mr-OPY2 physically interconnects with MAA_02467, the $M$. robertsii ortholog of STE50p (designated Mr-STE50), and thereafter the three MAPKKK proteins Mero-Ste11, Mero-Ssk2 and Mero-Bck1 (Fig. $5 \mathrm{~d}$ ). We disrupted $M r$-STE50 to produce $\Delta M r$ - STE50, which was complemented with its genomic clone to produce $C-\Delta M r$ STE50. Confirmation of gene disruption and mutant complementation is shown in Supplementary Fig. 2. $\Delta M r$-STE50 produced greyish green conidia compared with the darker green conidia of WT colonies (Fig. 5a). $\Delta M r$ - STE50 had reduced tolerance to high osmotic stress (Fig. 5b) and the cell wall-disturbing agent Congo Red (Supplementary Fig. 7), but its tolerance to 


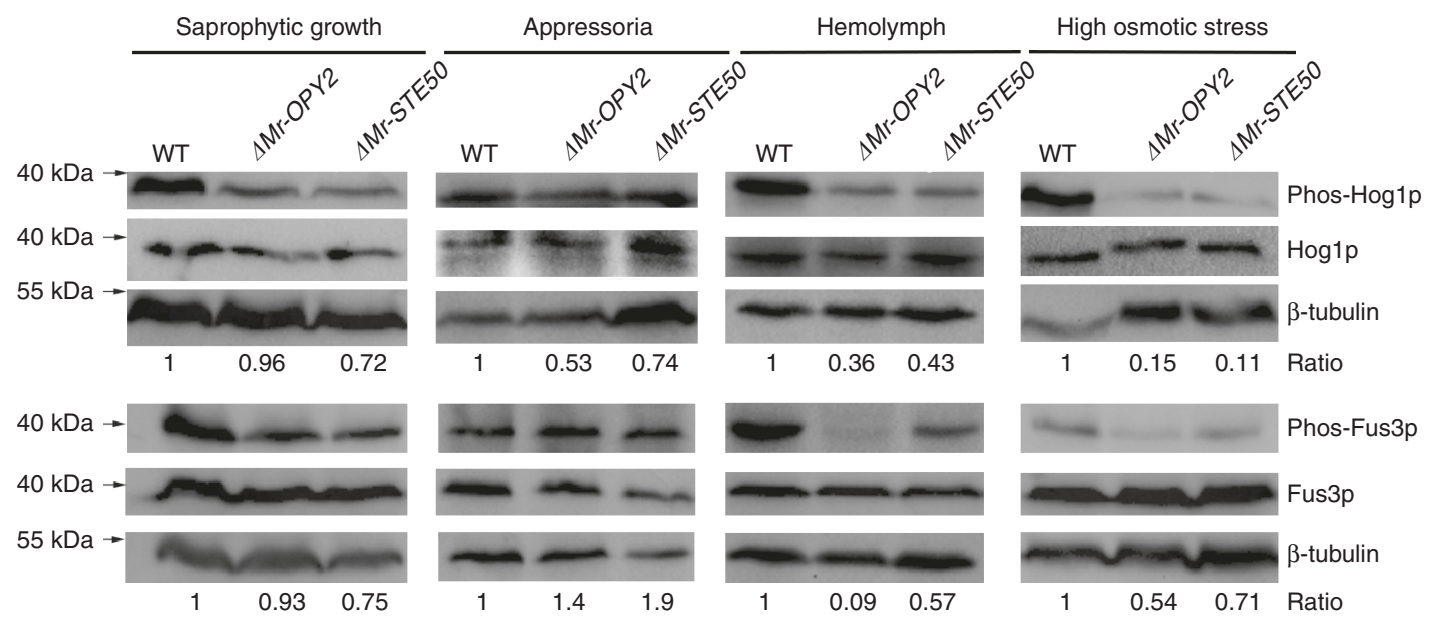

Fig. 6 Regulation of MAPK signaling pathways by Mr-OPY2 and Mr-STE50 during saprophytic growth, pathogenesis and under high osmotic stress. $\beta$-Tubulin was used as a loading control. Numbers indicate band intensity for Phos-Fus3p (Phos-Hog1p) relative to total Fus3p (Hog1p). WT values were set to 1. Images are representative of at least three independent experiments for each condition. The uncropped images of the western blots are shown in Supplementary Fig. 14

oxidative stress was unaltered (Supplementary Fig. 7). Like $\Delta M r$ OPY2, $\triangle M r$-STE50 did not produce appressoria, and was consequently unable to kill insects via topical application (Fig. 1a, c), but it showed WT virulence when injected into insects (Supplementary Fig. 3). Mycelia of WT and $\triangle M r-O P Y 2$ grew out of cadavers commencing 3 days after death. In contrast, no mycelium emerged on the $\Delta M r$-Ste50 infected insects, although cadavers contained mycelia (Fig. 1b). There were no significant $(P>0.05$, Tukey's test in one-way ANOVA) differences between the WT and $C-\Delta M r-S T E 50$ in any assays.

Previously, we found that the Fus3-MAPK and Hog1-MAPK signaling pathways regulate $M$. robertsii's infectivity and tolerance to high osmotic stress ${ }^{24}$. To investigate if Mr-OPY2 regulates these two MAPK signaling pathways we compared the phosphorylation level of Fus3-MAPK and Hog1-MAPK in WT, $\Delta M r$ OPY2 and $\triangle M r$-STE50 using their respective antibodies. During saprophytic growth in SDY, phosphorylation levels of Hog1MAPK and Fus3-MAPK were similar in $\triangle M r$-OPY2 and $\Delta M r$ STE50, and slightly lower than in the WT (Fig. 6). Phosphorylation of Fus3-MAPK and Hog1-MAPK was reduced in $\triangle M r-O P Y 2$ and $\triangle M r$-STE50 growing in hemolymph or under high osmotic stress (Fig. 6). Thus, in hemolymph, phosphorylation of Fus3MAPK was reduced 1.7 -fold and 11 -fold relative to WT in $\Delta M r$ STE50 and $\triangle M r-O P Y 2$, respectively. During appressorial formation, phosphorylation of Hog1-MAPK was reduced in the two mutants; whereas phosphorylation of Fus3-MAPK was unchanged compared wit the WT (Fig. 6).

Mr-OPY2 negatively regulates AFTF1 during cuticle penetration. RNA-seq was used to compare WT and $\triangle M r$-OPY2 transcriptomes of undifferentiating hyphae in SDY and hemolymph cultures, and germlings differentiating appressoria on locust wings. WT and $\triangle M r-O P Y 2$ grown in SDY, hemolymph and locust cuticle differed by 82,5 and 39 DEGs (differentially expressed genes), respectively. Only one DEG encoding a hypothetical protein was shared in the three growth conditions (Fig. 7a), indicating that Mr-OPY2 regulates distinctive subsets of genes during saprophytic growth, cuticle penetration and colonization of the hemocoel.

During differentiation of appressoria and cuticle penetration, a single transcription factor (MAA_08552) with a $\mathrm{Zn}_{2} \mathrm{Cys}_{6}$ fungal type DNA binding domain, designated as AFTF1, was differentially expressed between WT and $\triangle M r-O P Y 2$. qRT-PCR analysis showed Aftf1 was upregulated $\sim 31$ fold $(P<0.01$, Tukey's test in one-way ANOVA) on insect cuticle relative to hemolymph and SDY (Fig. 7b). On locust wings, expression of Aftf1 in $\triangle M r-O P Y 2$ was 7.2-fold higher than WT $(P<0.01$, Tukey's test in one-way ANOVA), but it was reduced $>11$-fold $(P<0.01$, Tukey's test in one-way ANOVA) in $\triangle M r$-STE50 and the two MAPK mutants (AMeor-Fus3 and $\Delta$ Mero-Slt2) (Fig. 7c). Both $\Delta$ Meor-Fus3 and $\Delta$ Mero-Slt2 are impaired in appressorial formation ${ }^{24}$.

In order to investigate the functions of $A f t f 1$, we deleted its ORF to produce $\Delta A f t f 1$, which was complemented with its genomic clone to produce $C-\Delta A f t f 1$. Confirmation of gene disruption and mutant complementation is shown in Supplementary Fig. 2. We also constructed strains (designated as $A f t f 1^{\mathrm{OE}}$ ) overexpressing $A f t f 1$ driven by the constitutive promoter (Ptef) of the translation elongation factor gene from Aureobasidium pullulans ${ }^{34}$. Germlings of two randomly selected Aftf1 $\mathrm{OE}$ transformants significantly $(P<0.01$, Tukey's test in one-way ANOVA) upregulated ( 300-fold) transcription of Aftf1 compared with WT when differentiating appressoria on locust cuticle (Supplementary Fig. 9a). No intra-strain differences were found between the two isolates in any assay, and so representative data for a single isolate is provided. Conidiation and saprophytic growth of $A f t f 1^{\mathrm{OE}}$ and $\Delta A f t f 1$ on $\mathrm{PDA}$ resembled the WT (Supplementary Fig. 9b, c). However, appressorial formation by $\Delta A f t f 1$, and to a lesser extent $A f t f 1{ }^{\mathrm{OE}}$, was significantly delayed ( $P$ $<0.05$, Tukey's test in one-way ANOVA) (Fig. $7 \mathrm{~d}$ ), significantly $(P<0.05$, Tukey's test in one-way ANOVA) reducing pathogenicity compared to the WT (Fig. 7e). In all the above assays, there were no significant $(P>0.05$, Tukey's test in one-way ANOVA) differences between the WT and C- $\Delta A f t f 1$ (Fig. 7 and Supplementary Fig. 9). Therefore, we conclude that the precise level of AFTF1 protein controlled by Mr-OPY2 is important for appressorial formation and pathogenicity.

Appressorial production is associated with synthesis of key effector proteins influencing virulence ${ }^{24}$. RNA-seq analysis showed that four cuticle-degrading proteases (MAA_00460, maa_07827, MAA_07828 and MAA_09637) were downregulated in $\triangle M r-O P Y 2$ during penetration of the cuticle. In addition, a cell wall surface protein Mas1 (MAA_08589) was upregulated; its ortholog is an important factor for appressorial formation in Magnaporthe oryzae ${ }^{35}$. qRT-PCR analysis showed that the protease MAA_09637 was also down-regulated in $\Delta A f t f 1$, suggesting that AFTF1 could regulate a subset of the genes 
a

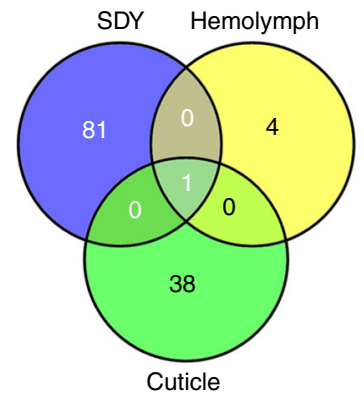

b

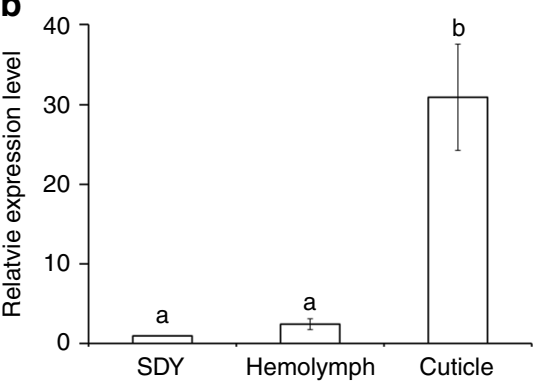

C

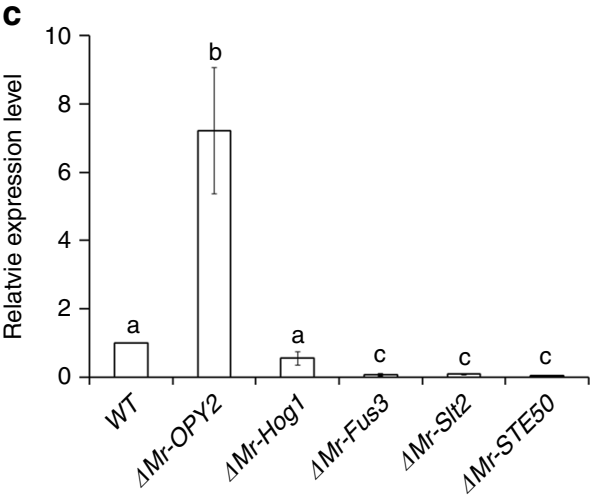

d

e
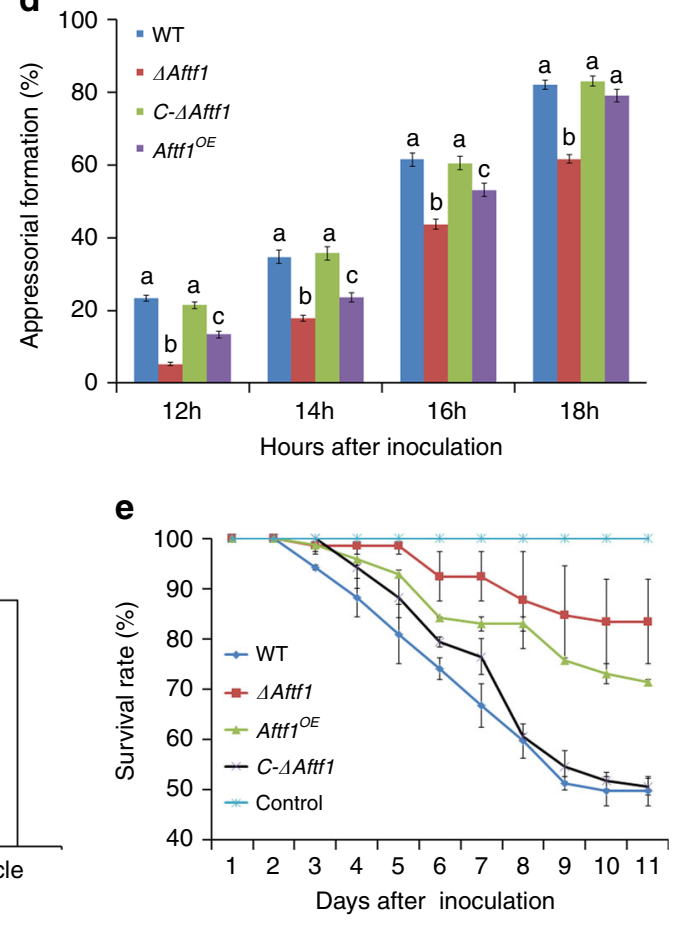

f

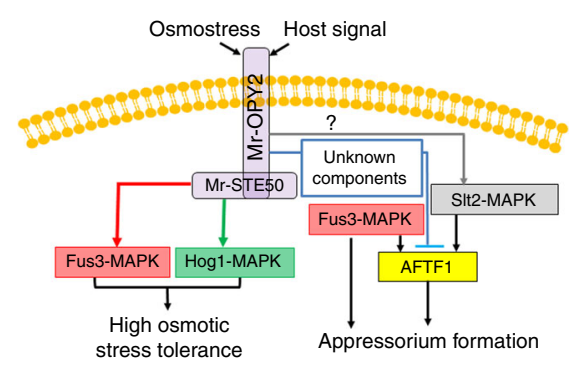

Fig. 7 Identification and characterization of the appressorial formation transcription factor AFTF1 and its negative regulation by Mr-OPY2. a Venn diagram of RNA-seq analysis showing the distribution of shared DEGs in transcriptomes of WT and $\triangle M r-O P Y 2$ during growth in SDY (SDY), the insect cuticle (Cuticle) and hemolymph (Hemolymph). Two biological repeats were established for each treatment. $\mathbf{b}$ qRT-PCR analysis of Aftf1 expression by the WT grown in hemolymph and on locust cuticle relative to expression during saprophytic growth in SDY (which is set to 1 in the figure). c qRT-PCR analysis of Aftf1 expression by the WT, $\triangle M r$-OPY2, $\triangle$ Mr-STE50, $\Delta$ Mero-Hog1, $\Delta$ Mero-Fus3 and $\Delta$ Mero-Slt2 during appressorial formation. The expression level in the WT is set to 1. The qRT-PCR analyses were repeated three times with three replicates per repeat. Data are expressed as the mean \pm SE. Values with different letters are significantly different $(n=3, P<0.05$, Tukey's test in one-way ANOVA). d The \% germlings differentiating appressoria on a hydrophobic plastic surface in the WT, $\triangle A f t f 1$ (the Aftf1 disruption mutant), C- $\Delta$ Aftf1 (the complemented strain of $\triangle A f t f 1$ ) and Aftf ${ }^{O E}$ (a strain overexpressing Aftf1). Appressorium formation assays were repeated three times with three replicates per repeat. Data are expressed as the mean $\pm \mathrm{SE}$. At each time point, values with different letters are significantly different $(n=3, P<0.05$, Tukey's test in one-way ANOVA). e Survival curves of G. mellonella larvae infected by WT, $\Delta$ Aftf1, C- $\Delta$ Aftf1 and $A f t f 1$ OE. Data are expressed as the mean \pm SE. Control: insects treated with $0.01 \%$ Triton $X-100$ solution. The bioassays were repeated three times with 40 insects per repeat. $f$ A schematic model of Mr-OPY2-mediated regulation of appressorial formation and osmotic stress tolerance. Mr-OPY2 regulates the phosphorylation level of Fus3 and Hog1-MAPK under high osmotic stress. During appressorial formation, Fus3-MAPK regulates the expression level of Aftf1, but regulation of Aftf1 by Mr-OPY2 is mediated by unidentified components. SIt2-MAPK also regulates Aftf1, but it remains to be determined whether Mr-OPY2 is involved in such regulation

regulated by Mr-OPY2 (Supplementary Fig. 10). The expression level of these five genes did not differ significantly $(P>0.05$, Tukey's test in one-way ANOVA) between WT and Atf1 ${ }^{\mathrm{OE}}$.

\section{Discussion}

Fungi can live as saprophytes in diverse habitats, on virtually any carbon source, or in commensal, mutualistic or parasitic relationships with plants, animals, insects or other fungi. The mechanisms that regulate transitions between saprophytic, symbiotic and pathogenic growth phases are poorly understood ${ }^{36}$, with the exception of a transcriptional regulator, Wor1/Ryp1, that governs lifestyle transitions in human pathogenic fungi, C. albicans and Histoplasma capsulatum ${ }^{37,38}$, and several plant pathogens including Fusarium verticillioides (Sge1) ${ }^{36}$. In this study, we report that the anchor membrane protein Mr-OPY2 is 
dispensable for saprophytic growth of $M$. robertsii, but is essential for this fungus to initiate development of infection structures (appressoria), which are a prerequisite for infection. Therefore, Mr-OPY2 regulates the transition of $M$. robertsii from saprophyte to pathogen; to our knowledge, this is the first demonstration that OPY2 regulates the initiation of infection in a pathogenic fungus. Homologs of Mr-OPY2 are conserved in plant and mammal pathogens, suggesting a potential role in regulating pathogenesis in other fungi, especially the many plant pathogenic fungi (e.g. $M$. oryzae) that also differentiate appressoria.

The mechanism by which Mr-OPY2 controls $M$. robertsii's saprophyte-to-pathogen transition involves precise regulation of its protein level, with higher levels during cuticular penetration than saprophytic growth. The Mr-OPY2 disruption mutant, and a manipulated strain (P404-Mr-OPY2) with a constantly low $\mathrm{Mr}$ OPY2 protein level, were both impaired in appressorial formation and thus in pathogenicity. This confirms that upregulation of the Mr-OPY2 protein level is necessary for infection processes. Conversely, a low level of Mr-OPY2 protein is important for normal saprophytic growth because forcefully elevating Mr-OPY2 (the strain P683-Mr-OPY2), with a constitutive promoter produced an abnormal colony phenotype with reduced conidial yield. P683-Mr-OPY2 was also impaired in appressorial formation, indicating that constitutively expressing high levels of Mr-OPY2 protein destabilizes appressorial formation. Mr-OPY2 is also upregulated in the insect hemolymph, but its role following penetration of the cuticle remains to be elucidated because $\Delta M r$ OPY2 resembled WT in its ability to colonize the hemocoel. Western blot analyses of Mr-OPY2 showed two bands in all strains (including WT) that express Mr-OPY2, which were absent in the deletion mutant of Mr-OPY2. One band is close to the theoretical molecular weight of Mr-OPY2 (45.5 kDa), whereas the other is $\sim 50 \mathrm{kDa}$. The heavier band could result from posttranslational modifications; the difference in the intensity of the two bands should result from the difference between the amount of protein modified and unmodified. C. albicans and S. cerevisiae OPY2 proteins were similarly bigger than their theoretical molecular weights due to glycosylation ${ }^{39,40}$.

The precise regulation of Mr-OPY2 protein levels is achieved via alternative transcription start sites. During pathogenesis, two Mr-OPY2 transcripts were produced; the abundant long transcript was inefficiently translated because its $5^{\prime}$ UTR has two small uORFs that were proven by two independent experiments to reduce translation efficiency of the downstream major ORF. As described in other studies ${ }^{29-31}$, small uORFs can modulate ribosomal access to the AUG start codon of the major ORF, decreasing its translation efficiency. Although the short transcript of $M r-O P Y 2$ has a lower copy number than the long one, it is more efficiently translated. Thus, although the long transcript was abundant during both saprophytic growth and pathogenesis, increased levels of the Mr-OPY2 protein during pathogenesis derived from the short transcript. We are not aware of any other reports on regulation of OPY2 protein levels via alternative transcription start sites. However, alternating transcription start sites is a common mechanism for regulating gene expression in fungi ${ }^{41}$.

Only a single novel transcription factor (AFTF1) is regulated (negatively) by Mr-OPY2 during cuticle penetration. Homologs of AFTF1 are also found in other fungi such as Fusarium oxysporum (EWY87630) and M. oryze (XP_003715433). While upregulation of AFTF1 is critical for appressorial formation, the process was delayed by overexpressing AFTF1. Therefore, a precisely modulated optimal level of AFTF1 is needed to choreograph appressorial formation with Fus3- and Slt2-MAPK upregulating Aftf1 during cuticle penetration and Mr-OPY2 ensuring that the level of AFTF1 is not too high for optimum effect. The precise regulation of Mr-OPY2 is presumably an adaptation to facilitate the precise regulation of AFTF1. The phosphorylation level of Fus3-MAPK is not controlled by $\mathrm{Mr}$ OPY2, suggesting that Mr-OPY2 does not regulate AFTF1 via Fus3-MAPK. Yeast two-hybrid assays showed that the MAPKKK (Bck1) of the Slt2-MAPK cascade interacts with Mr-STE50, which in turn interacts with Mr-OPY2, suggesting that Mr-OPY2 could regulate AFTF1 via Slt2-MAPK. Mr-OPY2 regulates phosphorylation of Hog1-MAPK during appressorial formation, but Hog1-MAPK does not regulate expression of $A f t f 1$ indicating that Mr-OPY2 has additional functions besides regulating AFTF1 (Fig. 7f).

In addition to regulating pathogenesis, OPY 2 is also necessary for $M$. robertsii to tolerate high osmotic stress, but as this stress did not alter Mr-OPY2 protein levels, low levels may be sufficient for stress resistance. Mr-OPY2 regulates $M$. robertsii's tolerance to high osmotic stress via the Hog1-MAPK and Fus3-MAPK pathways (Fig. 7f). S. cerevisiae Opy2 protein also mediates tolerance to high osmotic stress via Hog1-MAPK, but the Opy2 deletion mutant can grow under the osmotic stress because the redundant SSK branch also regulates Hog1-MAPK to tolerate osmotic stress ${ }^{26,40,42}$. The Opy2 protein of $C$. albicans is not involved in tolerance to high osmotic stress ${ }^{40}$, or C. albicans's pathogenicity against a natural host, but by an unknown mechanism does facilitate its ability to kill a non-natural host (the insect $G$. mellonella) following injection ${ }^{40}$. In contrast, we found that OPY2 is dispensable for hemocoel infection by the specialized insect pathogen $M$. robertsii. Therefore, the functions of OPY2 proteins have clearly diversified in fungi, along with their lifestyles. Our phylogenetic analysis confirmed that the OPY2 proteins from unicellular Ascomycota yeasts (including S. cerevisiae and $C$. albicans) are phylogenetically distant from their homologs in filamentous Ascomycota and Basidiomycota fungi.

In conclusion, we report that Mr-OPY2 controls the saprophyte-to-pathogen transition of the insect pathogen $M$. robertsii by negatively regulating the transcription factor AFTF1. This work describes a new circuit regulating fungal infection, and advances our insight into the mechanisms underpinning fungal lifestyle transitions.

\section{Methods}

Gene cloning and disruption. The flanking sequences of T-DNA were cloned by YADE (Y-shaped adaptor dependent extension) from $M$. robertsii mutants generated by T-DNA insertion as previously described ${ }^{22}$. M. robertsii ARSEF2575 and M. acridum ARSEF324 were obtained from the Agricultural Research Service Collection of Entomopathogenic Fungi. The primers used in this study are listed in Supplementary Table 2 .

Genes were disrupted based on homologous recombination using our previously developed high-throughput gene disruption methodology ${ }^{43}$. Disruption plasmids were constructed using Ppk2-OSCAR-GFP and pA-Bar, and confirmation of gene disruption was performed as described ${ }^{43}$. To complement a gene disruption mutant, the genomic clone of the gene containing the promoter region, ORF and termination region was amplified by PCR and inserted into the pPK2-Sur-GFP ${ }^{43}$. High fidelity Taq DNA polymerase (KOD Plus Neo, Osaka Japan) was used in PCR reactions to clone DNA fragments, which were confirmed by DNA sequencing. Agrobacterium tumefaciens AGL1 was used for fungal transformation as previously described ${ }^{44}$.

Histoimmunochemical staining and western blot analysis. In order to prepare an antiserum to Mr-OPY2, a predicted antigenic region (PGPNASPDQIKKHRD), residues $59-73$ of the $\mathrm{N}$-terminal domain, was synthesized and conjugated with keyhole limpet hemocyanin. The antibodies were raised in New Zealand White rabbits (Sigma, USA). Total cell membrane protein from fungal cells was prepared using the Mem-PER Eukaryotic Membrane Protein Extraction Reagent Kit (Thermo Scientific, USA), followed by purification with the Pierce sodium dodecyl sulfate polyacrylamide gel electrophoresis (SDS-PAGE) Sample Prep Kit (Thermo Scientific). Two identical SDS-PAGE gels were set up, one of which was used for western blot analysis, and the other one was stained with Coomassie Brilliant Blue in order to visualize the sample loading. The antiserum $\left(200 \mathrm{mg} \mathrm{ml}^{-1}\right)$ was used at a 1:500 dilution to detect Mr-OPY2 protein with western blot analysis. 
To analyze phosphorylation levels of Fus3 and Hog1-MAPK in M. robertsii, cell proteins were prepared with TCA (trichloroacetic acid) buffer as described ${ }^{45}$, and blotted to polyvinyldene difluoride membranes (Bio-Rad, USA). Rabbit antiphospho-p38 MAPK (Thr180/Tyr182) (Catalogue number: 9211), anti-phosphop44 MAPK (Catalogue number: 9101) and anti-p38 MAPK (Catalogue number: 9212) (Cell Signaling Technology, MA, USA) were used at a 1:1000 dilution to detect phosphorylation of Hog1-MAPK, Fus3-MAPK and the protein level of Hog1-MAPK, respectively. To prepare an antiserum to the Fus3-MAPK protein, a predicted antigenic region (CHDPEDEPTAPPTP) was synthesized, and the antiserum was raised in New Zealand White rabbit (HUABIO, Hangzhou, China). The antiserum $\left(0.4 \mathrm{mg} \mathrm{ml}^{-1} \mathrm{IgG}\right)$ was used at a 1:1000 dilution to detect the Fus3MAPK protein. $\beta$-tubulin was used as a loading control, which was detected using its mouse antibody (Catalogue number: M1305-2, HUABIO) at a 1:2000 dilution. Bound primary antibodies were revealed using horseradish peroxidase-conjugated goat anti-rabbit IgG (Catalogue number: HA1006, HUABIO) or goat anti-mouse IgG (Catalogue number: HA1006, HUABIO) with a 1:4000 dilution, and a chemiluminescence detection system (Clarity Western ECL, Bio-Rad).

Appressoria on the hydrophobic surface of a diagnostic slide (Live Focus, Jiangsu, China) were prepared for IIF as described ${ }^{46}$. Mr-OPY2 antiserum $(200 \mathrm{mg}$ $\mathrm{ml}^{-1}$ ) was diluted 200-fold for primary labeling, and FITC-conjugated-goat antirabbit IgG (Catalogue number: HA1004, HUABIO) was used for secondary labeling.

All Histoimmunochemical staining and Western blot analyses were repeated at least three times, and representative results are shown.

Assays of pathogenicity and conidiation and stress tolerance. Bioassays were conducted using last instar G. mellonella larvae (Ruiqingbait Co., Shanghai, China) by topical application or direct injections as described (Zhao et al., 2014). Tolerance to high osmotic stress, oxidative stress and cell wall-disturbing stress was assayed as previously described ${ }^{24}$. Determination of conidial yields and observation of conidiophores were conducted as previously described ${ }^{24}$. All assays were repeated three times.

Site mutagenesis. PCR mutagenesis was used to mutate the three AUGs into GUGs and thereby delete the uORFs in the 5'UTR of Mr-OPY2-L. The Mr-OPY2 genomic clone $(\mathrm{gMr} r \mathrm{OPY2})$ containing its exons, introns and terminator region was cloned by PCR using high fidelity Taq DNA polymerase into the pUCm-T (Takara, Dalian, China) to produce the plasmid PUC-gMr-OPY2. Two complementary primers (Supplementary Table 2) containing mutated sites flanked by 20 bases on each side were designed and used to amplify PUC-gMr-OPY2. The PCR product was then digested with DpnI (NEB, England) to cut the parental plasmid DNA, whereas the nascent PCR DNA was left intact. The PCR products were then incorporated into the E. coli strain that has an intact dam methylase system. The resulting plasmids thus contained no uORFs, and this was confirmed by DNA sequencing. The AUG at $-167 \mathrm{bp}$ ( $167 \mathrm{bp}$ upstream of the AUG of the major ORF) in $g M r$-OPY2 was initially mutated to produce $g M r-O P Y 2^{4 A U G 167}$, and the two linked AUGs at -363 bp and -366 bp in $g M r$-OPY ${ }^{\triangle A U G 167}$ were then mutated to form $g M r-O P Y 2^{\triangle A U G s}$.

Constructing strains with the Mr-OPY2 gene driven by Pgpd. To analyze the translation efficiency of the transcripts $M r-O P Y 2-L, M r-O P Y 2-S$ and $M r-O P Y 2-$ OPY2-L ${ }^{\triangle A U G s}$, each was placed downstream of the promoter Pgpd-NUTR (the $g p d$ gene's $5^{\prime} \mathrm{UTR}$ excluded) of the gpd gene from M. acridum ARSEF $324^{33}$. To do this, Pgpd-NUTR was amplified using the genomic DNA of M. acridum ARSEF324 as the template, which was then inserted into the NdeI/BamHI sites in the plasmid pBARGPE1 (obtained from Fungal Genetic Stock Center) to replace the Aspergillus nidulans gpd promoter, resulting in the plasmid pPgpd-NUTR. The DNA fragment containing Pgpd-NUTR and the TtrpC terminator was excised from pPgpd-NUTR with SpeI/SmaI and inserted into the SpeI/EcoRV sites of the plasmid pPK2-Sur$\mathrm{GFP}^{43}$ to produce the plasmid pPK2-Pgpd-NUTR.The transcripts of Mr-OPY2-L, $M r-O P Y 2-S$ and $M r$-OPY2-OPY2- $L^{\triangle A U G s}$ were cloned by PCR, digested with $E c o$ RI/SmaI and inserted into EcoRI/EcoRV sites downstream of the promoter Pgpd-NUTR in pPK2-Pgpd-NUTR to produce plasmids pPK2-Mr-OPY2-L, pPK2Mr-OPY2-S and pPK2-Mr-OPY2-OPY2-L ${ }^{\triangle A U G s}$, respectively. The three plasmids were then transferred into Agrobacterium tumefaciens and transformed into the $M r$-OPY2 deletion mutant $\triangle M r$-OPY2 to produce the strains $\triangle M r$-OPY2::Mr$O P Y 2-S, \triangle M r-O P Y 2:: M r-O P Y 2-L$ and $\triangle M r-O P Y 2:: M r-O P Y 2-L^{\triangle A U G s}$.

The strains $P 404-M r-O P Y 2$ and $P 683-M r-O P Y 2$ were constructed by placing the major ORF downstream of either the 404 bp promoter (P404) or the $683 \mathrm{bp}$ promoter (P683) of the M. acridum gpd gene (including the $5^{\prime} \mathrm{UTR}$ of the gpd gene $)^{33}$. The P404 and P683 promoters were cloned by PCR using the genomic DNA of M. acridum ARSEF324 as the template, and digested with enzymes NdeI/ $B a m H I$ to replace the $g p d$ promoter of $A$. nidulans in the plasmid pBARGPE1 and produce plasmids pP404 and pP683. The DNA fragment containing the promoter (P404 or P683) and trpC terminator region was excised with SpeI/SmaI and inserted into the SpeI/EcoRV sites in the pPK2-Sur-GFP plasmid to produce pPK2P404 and pPK-P683, respectively. The mORF of Mr-OPY2 was cloned by PCR and excised with EcoRI/KpnI, which was then inserted into the corresponding sites downstream of the promoters P404 and P683 in pPK2-P404 and pPK-P683 to form the plasmids pPK2-P404-Mr-OPY2 and pPK2-P683-Mr-OPY2, respectively.
pPK2-P404-Mr-OPY2 and pPK2-P683-Mr-OPY2 were then transferred into $A$. tumefaciens and transformed into the Mr-OPY2 deletion mutant $\triangle M r-O P Y 2$ to produce the strains $P 404-M r-O P Y 2$ and P683-Mr-OPY2.

Transcriptomic analysis using RNA-Seq. RNA-seq was used to profile transcriptomes during saprophytic growth in SDY, appressorial formation $(30 \mathrm{~h}$ post inoculation of conidia onto the hindwings of L. migratoria) and growth in B. mori hemolymph. Total RNA was extracted with TRIzol reagent (Life Technologies, USA). For saprophytic growth, conidia $\left(1 \times 10^{6}\right.$ conidia/ml $)$ were grown at $26^{\circ} \mathrm{C}$ for $36 \mathrm{~h}$ in SDY. For appressorial formation, hindwings of L. migrattoria manilensis were surface sterilized in $1 \%$ bleach as previously described ${ }^{21}$. A conidial suspension $\left(200 \mu \mathrm{l}\right.$ of $1 \times 10^{5}$ conidia/ml $)$ was applied to each hindwing which was then placed on a $1 \%$ water agar plate, and incubated at $26^{\circ} \mathrm{C}$ for $30 \mathrm{~h}$. For growth in the hemolymph, mycelium from a $36 \mathrm{~h}$ SDY culture was collected by filtration and washed with sterile water three times. One gram of mycelium was inoculated into $12 \mathrm{ml}$ of $B$. mori hemolymph and incubated for $12 \mathrm{~h}$ at $26^{\circ} \mathrm{C}$ with $100 \mathrm{rpm}$ shaking. The mycelium was then collected by filtration for RNA extraction. Locust wings and silkworm hemolymph were prepared as described ${ }^{24}$. Two biological repeats were established for each treatment.

Construction of libraries and sequencing with the Illumina HiSeq 2500 platform were performed by Novogene (Beijing, China). After paired-end sequencing, clean reads were obtained using the NGS QC Toolkit and mapped to the draft genome sequence of $M$. robertsii ARSEF $23^{16}$ using the programs TopHat 2.0.6 $6^{47}$. Reads that aligned uniquely to the reference sequence were used for gene expression quantification using the RPKM method ${ }^{48}$. Differential expression analysis was performed with DESeq software (version 1.18.0) ${ }^{49}$, and an adjusted $P$-value 0.05 (Benjamini-Hochberg method).

Construction of the strain overexpressing Aftf1. The strain overexpressing Aftfl was constructed by placing its ORF downstream of the constitutive promoter Ptef of the translation elongation factor gene from A. pullulans ${ }^{34}$. To do this, the promoter Ptef was cloned by PCR using the plasmid pPK2-Sur-GFP ${ }^{43}$ as the template, and digested with enzymes NdeI/BamHI to replace the gpd promoter of A. nidulans in the plasmid pBARGPE1 and produce the plasmid pPtef. The DNA fragment containing the promoter and trpC terminator region was excised with SpeI/SmaI and inserted into the SpeI/EcoRV sites in the pPK2-Sur-GFP plasmid to produce pPK2-Ptef. The ORF of Aftf1 was cloned by PCR and excised with EcoRI/ $E c o R V$, which was then inserted into the corresponding sites of pPK2-Ptef to produce pPK2-Ptef-Aftf1. pPK2-Ptef-Aftf1 was then transferred into A. tumefaciens and transformed into the WT M. robertsii. Overexpression of Aftfl was confirmed by qRT-PCR as described below.

Gene cloning and gene expression analysis. RLM RT-PCR kit was used to clone intact mRNA with a 7-methyl guanosine cap structure (Roche).

For quantitative RT-PCR, complementary DNAs (cDNAs) were synthesized with total RNAs (800 ng per reaction) with ReverTra AceqPCR RT Master Mix (Toyobo, Japan). Each cDNA was diluted 20 -fold, and used as a template for qRTPCR analysis using Thunderbird SYBR qPCR Mix without ROX (Toyobo). The genes act and tef were used as internal standards ${ }^{50}$. The relative expression level of each gene was determined using the $2^{-\Delta \Delta \mathrm{Ct}} \operatorname{method}^{51}$.

Northern blot analyses were conducted using standard methods ${ }^{52}$. The ORF of the Mr-OPY2 was used as the probe, which was labelled with DIG-DNA Labelling Kit (Roche). Northern blot and qRT-PCR analyses were repeated three times.

Yeast two-hybrid and autoactivation assays. Yeast competent cells were prepared using the Yeast-maker Yeast Transformation System 2 Kit (Clontech, USA), and yeast two-hybrid assays were conducted according to the manufacturer's instructions (Clontech). The ORFs of the tested genes were cloned by PCR and inserted into the plasmid pGADT7 (Mr-OPY2, Mero-BCK1, Mero-STE11 and Mero-SSK2) or pGBKT7 (Mr-STE50). The ORFs in the plasmids were confirmed by sequencing. The plasmid pGBKT7-Mr-STE50 was transformed into Y2HGold cells, and other plasmids (from pGADT7) were transformed into Y187 cells. After mating, the resulting strains were grown on the medium (SD-His-Ade-Leu-Trp) with $\mathrm{X}-\alpha$-gal and AbA (Aureobasidin A) (Takara). The autoactivation of $\mathrm{Mr}-$ STE50 in pGBKT7 was tested using Y2HGold cells. Yeast two-hybrid and autoactivation assays were repeated three times.

Statistical analysis. Tukey's honestly significant difference test in OriginPro 8.5 program was used in this study (OriginLab, USA)

Data availability. The nucleotide sequences of the long and short $M r-O P Y 2$ transcripts were deposited in the Genbank nucleotide database with accession codes KY548479 and KY548480. The RNA-seq data were deposited in the Genbank database with accession codes SRP105041. All other relevant data supporting the findings of the study are available in this article and its Supplementary Information files, or from the corresponding author upon request. 
Received: 19 May 2017 Accepted: 12 October 2017

Published online: 16 November 2017

\section{References}

1. Newton, A. C., Fitt, B. D. L., Atkins, S. D., Walters, D. R. \& Daniell, T. J. Pathogenesis, parasitism and mutualism in the trophic space of microbe-plant interactions. Trends. Microbiol. 18, 365-373 (2010).

2. O'Connell, R. J. et al. Lifestyle transitions in plant pathogenic Colletotrichum fungi deciphered by genome and transcriptome analyses. Nat. Genet. 44, 1060-1065 (2012)

3. Wang, L., Zhai, B. \& Lin, X. The link between morphotype transition and virulence in Cryptococcus neoformans. PLoS. Pathog. 8, e1002765 (2012).

4. Fesel, P. H. \& Zuccaro, A. Dissecting endophytic lifestyle along the parasitism/ mutualism continuum in Arabidopsis. Curr. Opin. Microbiol. 32, 103-112 (2016).

5. Barelli, L., Moonjely, S., Behie, S. W. \& Bidochka, M. J. Fungi with multifunctional lifestyles: endophytic insect pathogenic fungi. Plant Mol. Biol. 90, 657-664 (2016).

6. Hu, G. \& St Leger, R. J. Field studies using a recombinant mycoinsecticide (Metarhizium anisopliae) reveal that it is rhizosphere competent. Appl. Environ. Microbiol. 68, 6383-6387 (2002).

7. Fang, W. \& St Leger, R. J. Mrt, a gene unique to fungi, encodes an oligosaccharide transporter and facilitates rhizosphere competency in Metarhizium robertsii. Plant Physiol. 154, 1549-1557 (2010).

8. Pava-Ripoll, M. et al. The rhizosphere-competent entomopathogen Metarhizium anisopliae expresses a specific subset of genes in plant root exudate. Microbiology 157, 47-55 (2011).

9. Liao, X., O'Brien, T., Fang, W. \& St Leger, R. J. The plant beneficial effects of Metarhizium species correlate with their association with roots. Appl. Microbiol. Biotechnol. 98, 7089-7096 (2014).

10. Fang, W., Azimzadeh, P. \& St Leger, R. J. Strain improvement of fungal insecticides for controlling insect pests and vector-borne diseases. Curr. Opin. Microbiol. 15, 232-238 (2012).

11. Roberts, D. W. \& St Leger, R. J. Metarhizium spp. Cosmopolitan insectpathogenic fungi: Mycological aspects. Adv. Appl. Microbiol. 54, 1-70 (2004).

12. St Leger, R. J., Staples, R. C. \& Roberts, D. W. Cloning and regulatory analysis of starvation-stress gene, $s s g A$, encoding a hydrophobin-like protein from the entomopathogenic fungus, Metarhizium anisopliae. Gene 120, 119-124 (1992).

13. Wang, C. \& St Leger, R. J. The MAD1 adhesin of Metarhizium anisopliae links adhesion with blastospore production and virulence to insects, and the MAD2 adhesin enables attachment to plants. Eukaryot. Cell 6, 808-816 (2007).

14. Liu, R., Xu, C., Zhang, Q., Wang, S. \& Fang, W. Evolution of the chitin synthase gene family correlates with fungal morphogenesis and adaption to ecological niches. Sci. Rep. 7, 44527 (2017).

15. Bagga, S., Hu, G., Screen, S. E. \& St Leger, R. J. Reconstructing the diversification of subtilisins in the pathogenic fungus Metarhizium anisopliae. Gene 324, 159-169 (2004).

16. Gao, Q. et al. Genome sequencing and comparative transcriptomics of the model entomopathogenic fungi Metarhizium anisopliae and M. acridum. PLoS Genet. 7, e1001264 (2011).

17. Wang, C. \& St Leger, R. J. A collagenous protective coat enables Metarhizium anisopliae to evade insect immune responses. Proc. Natl Acad. Sci. USA 103, 6647-6652 (2006).

18. Fang, W. \& St Leger, R. J. RNA binding proteins mediate the ability of a fungus to adapt to the cold. Environ. Microbiol. 12, 810-820 (2010).

19. Fang, W., Fernandes, E. K., Roberts, D. W., Bidochka, M. J. \& St Leger, R. J. A laccase exclusively expressed by Metarhizium anisopliae during isotropic growth is involved in pigmentation, tolerance to abiotic stresses and virulence. Fungal Genet. Biol. 47, 602-607 (2010).

20. Wang, B., Kang, Q., Lu, Y., Bai, L. \& Wang, C. Unveiling the biosynthetic puzzle of destruxins in Metarhizium species. Proc. Natl Acad. Sci. USA 109, 1287-1292 (2012).

21. Zhao, H. et al. Host-to-pathogen gene transfer facilitated infection of insects by a pathogenic fungus. PLoS Pathog. 10, e1004009 (2014).

22. Zeng, G. et al. Genome-wide identification of pathogenicity, conidiation and colony sectorization genes in Metarhizium robertsii. Environ. Microbiol. 19, 3896-3908 (2017).

23. Fang, W., Pava-ripoll, M., Wang, S. \& St Leger, R. J. Protein kinase A regulates production of virulence determinants by the entomopathogenic fungus, Metarhizium anisopliae. Fungal Genet. Biol. 46, 277-285 (2009).

24. Chen, X. et al. MAPK cascade-mediated regulation of pathogenicity, conidiation and tolerance to abiotic stresses in the entomopathogenic fungus Metarhizium robertsii. Environ. Microbiol. 18, 1048-1062 (2016).

25. Krogh, A., Larsson, B., von Heijne, G. \& Sonnhammer, E. L. L. Predicting transmembrane protein topology with a hidden Markov model: application to complete genomes. J. Mol. Biol. 305, 567-580 (2001).
26. Yamamoto, K., Tatebayashi, K., Tanaka, K. \& Saito, H. Dynamic control of yeast MAP Kinase network by induced association and dissociation between the Ste50 scaffold and the Opy2 membrane anchor. Mol. Cell 40, 87-98 (2010).

27. Shimodaira, H. \& Hasegawa, M. CONSEL: for assessing the confidence of phylogenetic tree selection. Bioinformatics 1712, 1246-124 (2001).

28. St Leger, R. J., Butt, T., Goettel, M. S., Staples, R. C. \& Roberts, D. W. Production in vitro of appressoria by the entomopathogenic fungus Metarhizium anisopliae. Exp. Mycol. 13, 274-288 (1989).

29. Morris, D. R. \& Geballe, A. P. Upstream open reading frames as regulators of mRNA translation. Mol. Cell Biol. 20, 8635-8642 (2000).

30. Vilela, C. \& McCarthy, J. E. Regulation of fungal gene expression via short open reading frames in the mRNA $5^{\prime}$ untranslated region. Mol. Microbiol. 49, 859-867 (2003).

31. Onofre, C., Tome, F., Barbosa, C., Silva, A. L. \& Romao, L. Expression of human hemojuvelin (HJV) is tightly regulated by two upstream open reading frames in HJV mRNA that respond to iron overload in hepatic cells. Mol. Cell Biol. 35, 1376-1389 (2015).

32. Kos, M., Denger, S., Reid, G. \& Gannon, F. Upstream open reading frames regulate the translation of the multiple mRNA variants of the estrogen receptor alpha. J. Biol. Chem. 277, 37131-37138 (2002).

33. Cao, Y., Jiao, R. \& Xia, Y. A strong promoter, PMagpd, provides a tool for high gene expression in entomopathogenic fungus, Metarhizium acridum. Biotechnol. Lett. 34, 557-562 (2012).

34. Spear, R. N., Cullen, D. \& Andrews, J. H. Fluorescent label, confocal microscopy, and quantitative image analysis in study of fungal biology. Methods. Enzymol. 307, 607-623 (1999).

35. Xue, C. et al. Two novel fungal virulence genes specifically expressed in appressoria of the rice blast fungus. Plant. Cell 14, 2107-2119 (2002).

36. Brown, D. W., Busman, M. \& Proctor, R. H. Fusarium verticillioides SGElis required for full virulence and regulates expression of protein effector and secondary metabolite biosynthetic genes. MPMI 27, 809-823 (2014).

37. Huang, G. et al. Bistable expression of WOR1, a master regulator of white-opaque switching in Candida albicans. Proc. Natl Acad. Sci. USA 103, 12813-12818 (2006).

38. Nguyen, V. Q. \& Sil, A. Temperature-induced switch to the pathogenic yeast form of Histoplasma capsulatum requires Ryp1, a conserved transcriptional regulator. Proc. Natl Acad. Sci. USA 105, 4880-4885 (2008).

39. Yang, H. Y., Tatebayashi, K., Yamamoto, K. \& Saito, H. Glycosylation defects activate filamentous growth Kss1 MAPK and inhibit osmoregulatory Hog1 MAPK. EMBO. J. 28, 1380-1391 (2009).

40. de Dios, C., Román, E., Diez, C., Alonso-Monge, R. \& Pla, J. The transmembrane protein Opy2 mediates activation of the Cek1 MAP kinase in Candida albicans. Fungal. Genet. Biol. 50, 21-32 (2013).

41. Rojas-Duran, M. F. \& Gilbert, W. V. Alternative transcription start site selection leads to large differences in translation activity in yeast. RNA 18, 2299-2305 (2012).

42. Wu, C. L., Jansen, L. C., Zhang, D. Y. \& Whiteway, T. M. Adaptor protein Ste50p links the Ste11p MEKK to the HOG pathway through plasma membrane association. Genes Dev. 20, 734-746 (2006).

43. Xu, C. et al. A high-throughput gene disruption methodology for the entomopathogenic fungus Metarhizium robertsii. PLoS ONE 9, e107657 (2014),

44. Fang, W., Pei, Y. \& Bidochka, M. J. Transformation of Metarhizium anisopliae mediated by Agrobacterium tumefaciens. Can. J. Microbiol. 52, 623-626 (2006).

45. Foiani, M., Liberi, G., Piatti, S. \& Plevani, P. in Eukaryotic DNA Replication. A Practical Approach (ed. Cotterill, S.) 185-200 (Oxford University Press, UK, 1999).

46. Pemberton, L. F. in Yeast genetics: methods and protocols in molecular biology (eds Smith, J. S. \& Burke, D. J.) 79-90 (Springer Science, Humana Press, NY, 2014).

47. Trapnell, C., Pachter, L. \& Salzberg, S. L. TopHat: discovering splice junctions with RNA-Seq. Bioinformatics 25, 1105-1111 (2009).

48. Mortazavi, A., Williams, B. A., McCue, K., Schaeffer, L. \& Wold, B. Mapping and quantifying mammalian transcriptomes by RNA-Seq. Nat. Methods $\mathbf{5}$, 621-628 (2008).

49. Ostlund, G. et al. InParanoid 7: new algorithms and tools for eukaryotic orthology analysis. Nucleic Acids Res. 38, D196-D203 (2010).

50. Fang, W. \& Bidochka, M. J. Expression of genes involved in germination, conidiogenesis and pathogenesis in Metarhizium anisopliae using quantitative real-time RT-PCR. Mycol. Res. 110, 1165-1171 (2006).

51. Livak, K. J. \& Schmittgen, T. D. Analysis of relative gene expression data using real-time quantitative PCR and the $2^{-\Delta \Delta} \mathrm{Ct}$ method. Methods 25, 402-408 (2001).

52. in Molecular cloning: a laboratory manual (eds Sambrook, J., Fritsch, E. F. \& Maniatis, T.). (Cold Spring Harbor Laboratory Press, NY, 2001). 


\section{Acknowledgements}

This work was funded by the National Natural Science Foundation of China (31272097 and 31672078)

\section{Author contributions}

N.G. and Y.Q. designed and performed experiments. X.C. constructed the deletion mutant of Aftf1. Q.Z. performed the phylogenetic analysis. G.Z., X.Z. and W.M.

performed complementation of the deletion mutants and bioassays. C.X. analysed the

RNA-seq data. R.J.S.L. wrote the manuscript. W.F. conceived the idea, designed and

coordinated the study, and wrote the paper.

\section{Additional information}

Supplementary Information accompanies this paper at doi:10.1038/s41467-017-01756-1.

Competing interests: The authors declare no competing financial interests.

Reprints and permission information is available online at http://npg.nature.com/ reprintsandpermissions/
Publisher's note: Springer Nature remains neutral with regard to jurisdictional claims in published maps and institutional affiliations.

(c) (i) Open Access This article is licensed under a Creative Commons Attribution 4.0 International License, which permits use, sharing, adaptation, distribution and reproduction in any medium or format, as long as you give appropriate credit to the original author(s) and the source, provide a link to the Creative Commons license, and indicate if changes were made. The images or other third party material in this article are included in the article's Creative Commons license, unless indicated otherwise in a credit line to the material. If material is not included in the article's Creative Commons license and your intended use is not permitted by statutory regulation or exceeds the permitted use, you will need to obtain permission directly from the copyright holder. To view a copy of this license, visit http://creativecommons.org/ licenses/by/4.0/.

(C) The Author(s) 2017 